\title{
Effects of Network Structure, Competition and Memory Time on Social Spreading Phenomena
}

\author{
James P. Gleeson, ${ }^{1, *}$ Kevin P. O’Sullivan, ${ }^{1}$ Raquel A. Baños, ${ }^{2}$ and Yamir Moreno, ${ }^{2,3}$ \\ ${ }^{1}$ MACSI, Department of Mathematics and Statistics, University of Limerick, Ireland \\ ${ }^{2}$ Instituto de Biocomputación y Física de Sistemas Complejos (BIFI), Universidad de Zaragoza, \\ Mariano Esquillor s/n, 50018 Zaragoza, Spain \\ ${ }^{3}$ Department of Theoretical Physics, Faculty of Sciences, University of Zaragoza, Zaragoza 50009, Spain \\ and Institute for Scientific Interchange (ISI), Turin, Italy
}

(Received 1 December 2015; revised manuscript received 29 February 2016; published 13 May 2016)

\begin{abstract}
Online social media has greatly affected the way in which we communicate with each other. However, little is known about what fundamental mechanisms drive dynamical information flow in online social systems. Here, we introduce a generative model for online sharing behavior that is analytically tractable and that can reproduce several characteristics of empirical micro-blogging data on hashtag usage, such as (timedependent) heavy-tailed distributions of meme popularity. The presented framework constitutes a null model for social spreading phenomena that, in contrast to purely empirical studies or simulation-based models, clearly distinguishes the roles of two distinct factors affecting meme popularity: the memory time of users and the connectivity structure of the social network.
\end{abstract}

DOI: 10.1103/PhysRevX.6.021019

\section{INTRODUCTION}

Recent advances in communication technologies and the emergence of social media have made it possible to communicate rapidly on a global scale. However, since we receive pieces of information from multiple sources, this has also made the information ecosystem highly competitive; in fact, users' influence and visibility are highly heterogeneous, and topics strive for users' attention in online social systems. Although several studies have described the dynamics of information flow in popular communication media [1-5], the main factors determining the observed patterns have not been identified, and there is no theoretical framework that addresses this challenge. Indeed, given the potential for applications-e.g., having more efficient systems to spread information for safety and preparedness in the face of threats-a better understanding of how memes (ideas, hashtags, etc.) emerge and compete in online social networks is critical.

Information often spreads through a social network as a cascade: A person adopts a new behavior, installs a new app, or sends a news item or rumor to his friends (e.g., by tweeting it on Twitter). The avalanche spreads if the friends decide to also adopt the new behavior and in turn pass on the social influence effect to their own friends, who may further propagate the behavior. Following the usage in the

*james.gleeson@ul.ie

Published by the American Physical Society under the terms of the Creative Commons Attribution 3.0 License. Further distribution of this work must maintain attribution to the author(s) and the published article's title, journal citation, and DOI.
Subject Areas: Complex Systems

review [6], we apply the term "social spreading phenomena" to describe such cascading or "viral" propagation [7]. The latter term is used because the description of information spreading bears some similarity to epidemics of contagious disease; the effects of network structure on disease contagion have been well studied by physicists [8] (see Ref. [9] for a recent review). However, unlike epidemics of a single disease strain, we focus on social spreading phenomena that occur in the presence of competition between a large number of different items of similar type. Examples of the types of items include URLs on Twitter [1,2], apps on Facebook [5,10], or videos on YouTube [11]. In each of these examples, users make choices-often influenced by the choices they have seen their friends make - and the accumulation of many choices leads to a distribution of popularity of the items: Some items become extremely popular, while other items remain obscure.

To enable a succinct general description, we use Dawkins's term [12] "memes" because they are all "elements of a culture or system of behavior passed from one individual to another by imitation..." [13]. Note that we do not restrict our study only to very popular memes; indeed, our interest is in understanding the entire popularity distributions of memes, from the unpopular to the very popular. This definition of a meme has also been used by researchers studying cascades on Facebook [14], the spreading of news through blogs [15], and the popularity of hashtags on Twitter [3,16], but it can also be applied to analyze popularity distributions of offline items (where copying promotes spreading) such as baby names [17], dog breeds [18], and even citations (which are a type of 
popularity measure) of scientific papers $[19,20]$. The memes in these examples are all relatively simple units of information that are easily identified in data sets; recent work has also demonstrated that more complex memes (represented by the appearance of common phrases, such as "quantum" or "graphene," in the scientific literature) can be recognized by their inheritance patterns in the citation network [21].

A notable characteristic of many meme popularity distributions is that they are very fat-tailed: If a powerlaw distribution is fitted to the data, then the power-law exponent $\tau$ is typically between 1.5 and 2, which lies outside the range of exponents produced by models of cumulative-advantage [22-24] or preferential-attachment [25] type. The statistical physics of avalanches has been studied in the context of condensed-matter systems, where the flip of a single magnetic spin domain can cause its neighboring domains to also flip and thus initiate a cascade [26]. If the physical parameters of such a system are tuned to place it at a critical point [27], the sizes of avalanches are power-law distributed; the sandpile model of self-organized criticality (SOC) self-tunes so that the system balances at the critical point [28]. However, unlike the memoryless particles or magnetic spins that constitute the microscopic entities in condensed-matter avalanches, humans absorb and transmit information on a wide variety of time scales that range from seconds to weeks [29,30]. Models of social interaction must therefore include "memory" effects (nonMarkovian aspects) that lead to the emergence of characteristics that are qualitatively different from those seen in condensed-matter avalanches. The non-Markovian aspects of human temporal behavior have attracted considerable recent attention (e.g., Refs. [31-35]), but we wish to investigate the effects of memory on popularity avalanches caused by users choosing between multiple items that they have seen in the past.

To address this problem, we develop a theoretical framework that models how users choose among multiple sources of incoming information and affect the spreading of memes on a directed social network, like Twitter [1-3]. Our probabilistic model, in contrast to other studies $[3-5,19,36]$ that use intensive computational simulations to fit to data, allows us to get analytical insights into the respective roles of the network degree distribution, the memory-time distribution of users, and the competition between memes for the limited resource of user attention. The model is a "null model" in the sense that it is analytically tractable, yet realistic enough to be fitted to empirical data and to reproduce some important characteristics of the data. We show that fitting to time-dependent data requires a nontrivial memory-time distribution, which is not possible with the toy model of Ref. [37], where users can remember only one meme. However, the phenomenon of "competitioninduced criticality" that was first identified in Ref. [37] is shown to be robust to the inclusion of memory times, heterogeneous user activity rates, and complex network structures in the more realistic model used here. The current model requires more sophisticated mathematical analysis than that of Ref. [37] to deal with the long memory of users, but it enables us to understand how heavy-tailed distributions of meme popularity evolve over a range of time scales, as a few memes "go viral" but the majority become only moderately popular.

We phrase the model in terms of meme propagation on a directed social network (like Twitter) and interpret a "meme" to be any distinct piece of information that is easily copied and transmitted (e.g., a hashtag or URL within a tweet). However, it should be clear that the model and its results can also be extended to the other examples of viral phenomena discussed above. For the adoption of apps on Facebook, for example, the memes are the notifications sent when a user installs an app [10]. If a friend is prompted by this notification to also install the app, then the meme propagates on the network and its popularity is measured by the number of installations of the app. We show that the crucial property of the model that poises the system at criticality is the competitive pressure for the limited resource of user attention, and this property is common to a broad range of social spreading phenomena that are characterized by the availability of large time-dependent data sets.

The remainder of the paper is structured as follows. The model is introduced in Sec. II; in Secs. III and IV, we derive and analyze a branching-process description of the model dynamics. We confirm the results of this analysis using numerical simulations in Sec. V and then use the analytical results to fit the model to hashtag popularities extracted from micro-blogging data in Sec. VI, and to explain novel features of the time-dependent data. In Sec. VII, we discuss limitations of the model and possible extensions of it. Note that Secs. III and IV may be omitted on a first reading without affecting the understanding of the model and the main results.

\section{MODEL}

In online communication platforms like Twitter, users follow (receive the broadcasts or "tweets" of) other users. In graph-theoretical terms, these relationships constitute directed links from the followed node (user) to the follower (Fig. 1). The network structure is defined by the joint probability $p_{j k}$ that a randomly chosen node (user) has indegree $j$ (i.e., follows $j$ other Twitter users) and out-degree $k$ (i.e., has $k$ followers), but the network is otherwise assumed to be maximally random (a configuration model directed network). The mean degree of the network is $z=\sum_{j, k} k p_{j k}=\sum_{j, k} j p_{j k}$. If we simplify the model by assuming that all users follow $z$ others-as we sometimes do to highlight the role of the out-degree distribution - then $p_{j k}$ can be replaced with $\delta_{j, z} p_{k}$, where $\delta_{j, z}$ is the Kronecker delta and $p_{k}$ is the out-degree distribution. 


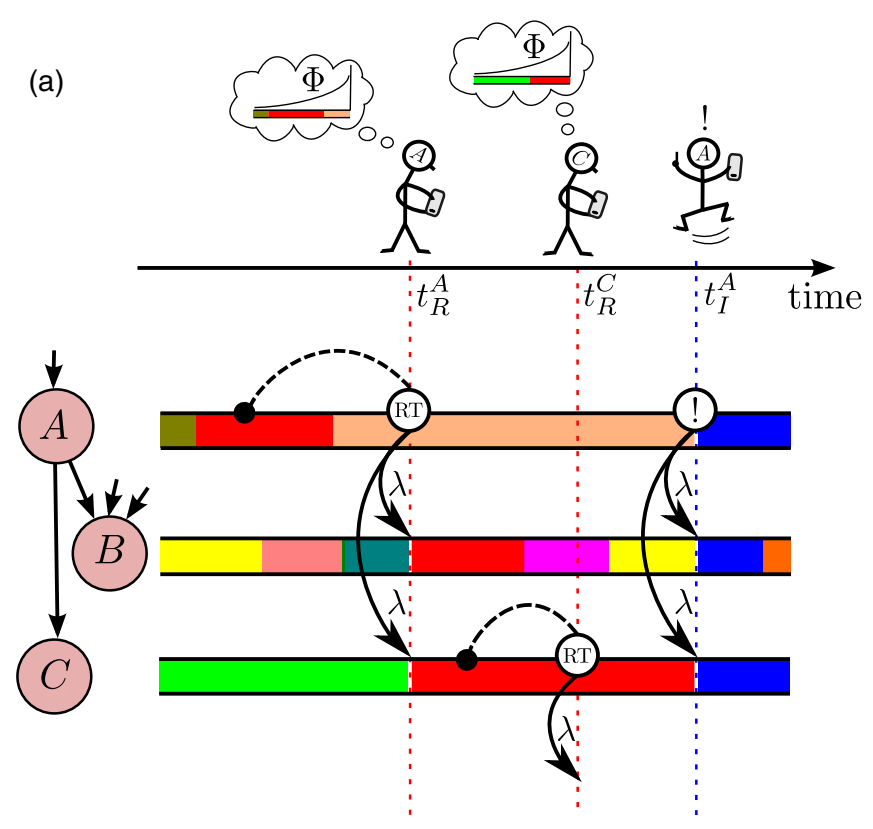

(b)

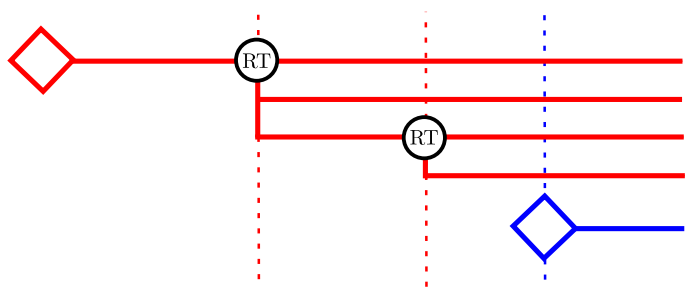

FIG. 1. Schematic of the model. (a) Timeline of users' actions in a typical realization of the model. User $A$ is followed by users $B$ and $C$; arrows between nodes denote the direction of information transmission. Note that user $B$ also follows many other users, and so his stream contains more memes than the streams of $A$ or $C$. At time $t_{R}^{A}$, user $A$ retweets a previously seen meme (with probability $1-\mu$, given $A$ is active). She chooses the red meme to retweet, by looking backwards in her stream a distance determined by the memory-time distribution $\Phi$ (only memes that $A$ deemed "interesting" are shown in her stream). Her retweet of the red meme is accepted as interesting (and so inserted into their stream) by each follower of $A$ with probability $\lambda$. At time $t_{R}^{C}$, user $C$ retweets the red meme to his followers, thus further increasing the popularity of the red meme. At time $t_{I}^{A}$, user $A$ innovates (a probability $\mu$ event, given $A$ is active) by inventing the new blue meme and broadcasting it to her followers. (b) Branching process representation (Sec. III) of the popularities of the red meme and of the blue meme. Each retweet generates new branches of the process, as the meme is inserted into the streams of followers of the tweeting user.

Each user has a "stream" that records all tweets received by the user, time stamped by their arrival time. We assume that only a fraction $\lambda$ of the tweets received are deemed "interesting" by the user, and only the interesting tweets are considered for possible retweeting by that user. (Here, we use the term "retweeting" in a general sense, to include any reuse of a previously received meme such as a hashtag. Note that a meme may be retweeted more than once by a user, unlike the model of Ref. [35]). The activity rate of a user- the average number of tweets that she sends per unit time, i.e., the rate of the Poisson process that describes her tweeting activity - can depend on how well connected the user is within the social network [3], and we assume it depends on her in-degree $j$ and out-degree $k$ [her " $(j, k)$ class" for short]; this assumption is supported by empirical evidence from Twitter (see Fig. 6 of Ref. [38]). The user activity rates $\beta_{j k}$ give the relative activity levels of users in the $(j, k)$ class; the rates are normalized by choosing time units so that $\sum_{j k} \beta_{j k} p_{j k}=1$. If there are $N$ users in the network, this rate implies that an average of $N$ tweets are sent in each model time unit. To simplify the analysis, we sometimes specialize to the case where all user activity rates are equal: $\beta_{j k}=1$.

When a user decides, at time $t$, to send a tweet, she has two options (see Fig. 1): With probability $\mu$, the user innovates, i.e., invents a new meme, and tweets this new meme to all her followers. The new meme appears in the user's own stream (it is automatically interesting to the originating user), and in the streams of all her followers (where it may be deemed interesting by each follower, independently, with probability $\lambda$ ). If not innovating (with probability $1-\mu$ ), the user instead chooses a meme from her stream to retweet. The meme for retweeting is chosen by looking backwards in time an amount $t_{m}$ determined by a draw from the memory-time distribution $\Phi\left(t_{m}\right)$ and finding the first interesting meme in her stream that arrived prior to the time $t-t_{m}$. The retweeted meme then appears in the streams of the user's followers (time stamped as time $t$ ), but because it is a retweet, it does not appear a second time in the stream of the tweeting user. The popularity $n(a)$ of a meme is the total number of times it has been tweeted or retweeted by age $a$, i.e., by a time $a$ after its first appearance (when it was tweeted as an innovation) [19]. Figure 2 shows some examples of evolving meme popularities: each panel displays the popularity $n(a)$ of a single meme as a function of its age $a$.

The model as described is a "neutral model" [39,40] in the sense that all memes have the same "fitness" [41]: No meme has an inherent advantage in terms of its attractiveness to users. Nevertheless, the competition between memes for the limited resource of user attention causes initial random fluctuations in popularities of memes to be amplified and leads to the variability across memes seen in Fig. 2 and to popularity distributions with very heavy tails [17]: heavier, for example, than can be generated by models of preferential attachment or cumulative advantage type $[22,23,25,42,43]$. This "competition-induced criticality" was studied for a zero-memory $\left[\Phi\left(t_{m}\right)=\delta\left(t_{m}\right)\right]$ version of this model in Ref. [37]. Indeed, the results of Ref. [37] can be obtained as a special case of the model described here, by setting $\Phi\left(t_{m}\right)=\delta\left(t_{m}\right), \lambda=1, \quad \beta_{j k} \equiv 1$, and $p_{j k}=\delta_{j z} p_{k}$; numerical simulation results for a closely related model were first reported in Ref. [3]. 

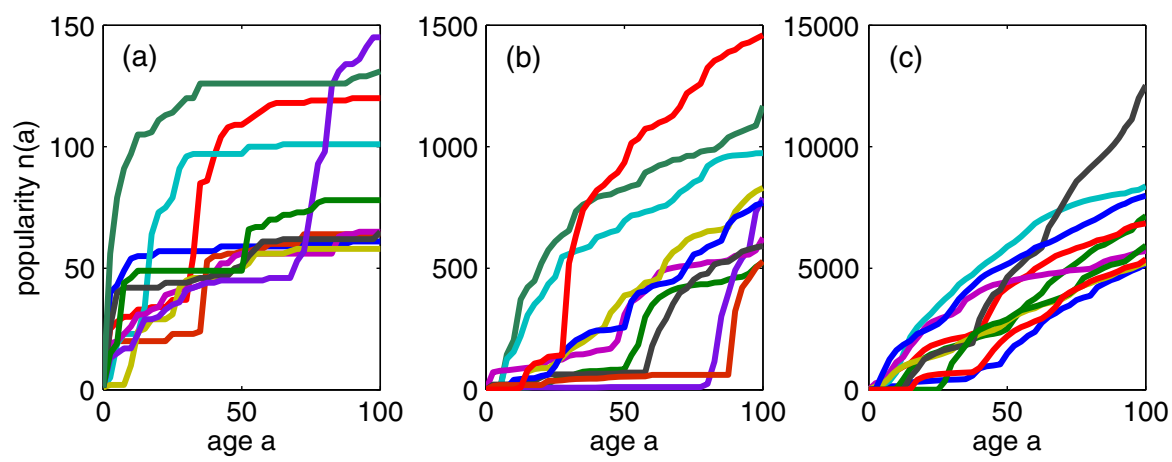

FIG. 2. Examples of the age dependence of meme popularity from numerical simulations of the model. Each panel shows the popularity of 10 different memes; the memes plotted are chosen at random from those whose popularity at age 100 is of order (a) $10^{2}$, (b) $10^{3}$, or (c) $10^{4}$. For model parameters, see the caption of Fig. 4(a).

A branching process approximation [35,44] for the model enables us to understand how the network structure (via the out-degree distribution $p_{k}$ ) and the users' memorytime distribution $\left[\Phi\left(t_{m}\right)\right]$ affect the popularity distribution of memes. Defining $q_{n}(a)$ as the probability that a meme has popularity [total number of (re)tweets] $n$ at age $a$, the branching process provides analytical expressions that determine the probability generating function (PGF) $[45,46]$ of the popularity distribution,

$$
H(a ; x)=\sum_{n=1}^{\infty} q_{n}(a) x^{n}
$$

The details of the derivation and analysis of the branchingprocess approximation are given in Secs. III and IV. The reader who is mainly interested in the applications of the model may jump straight to Sec. V, while noting that the most important outcome of the analysis is that in the smallinnovation limit $\mu \rightarrow 0$, the model describes a critical branching process, with power-law distributions of popularity (avalanche size) [47-50].

\section{DERIVATION OF BRANCHING PROCESS APPROXIMATION}

\section{A. Derivation of governing equations}

We define $G_{j k}(\tau, \Omega ; x)$ as the probability generating function for the size of the "retweet tree," as observed at time $\Omega$, that grows from the retweeting of a meme that entered, at time $\tau \leq \Omega$, the stream of a $(j, k)$-class user [see Fig. 3(b)]. To obtain an equation for $G_{j k}$, we consider the stream of a random $(j, k)$-class user (called "user $A$ ") with a

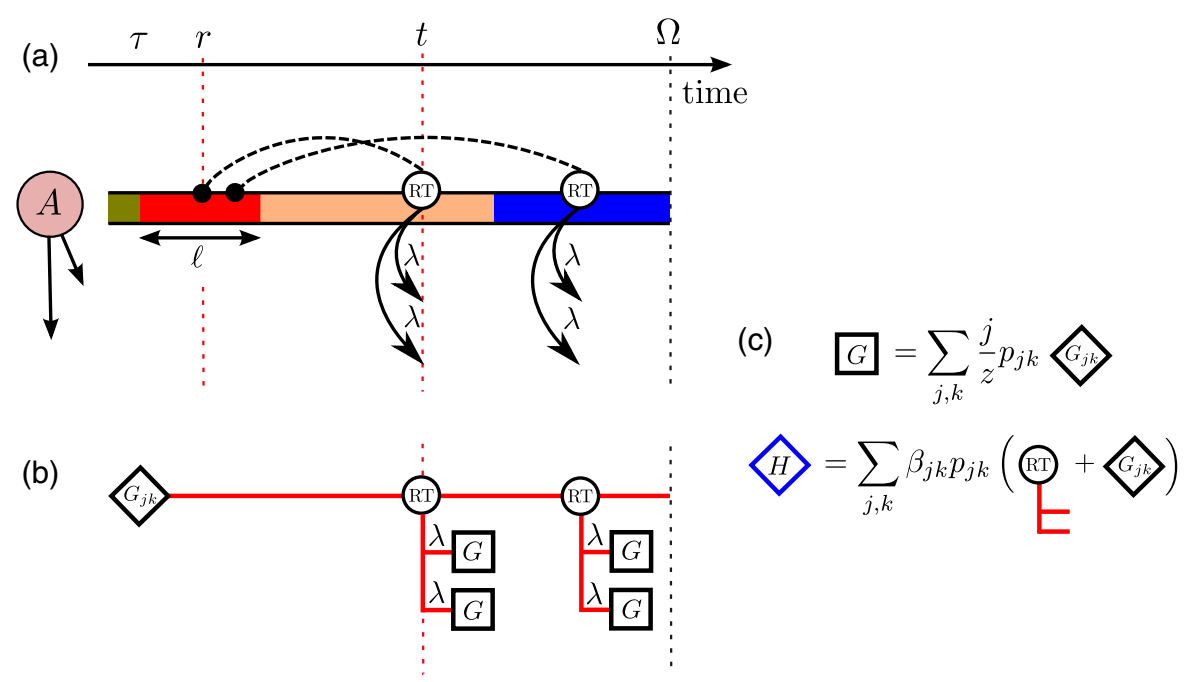

FIG. 3. Schematic for the derivation of the PGF equations (see Sec. III). (a) The stream of user $A$, showing only memes that were deemed interesting by user $A$. Each color represents a different meme. At time $t$, user $A$ decides to retweet a meme from the past and looks back to time $r$, where she finds meme $M$ (colored red). She sends this meme to her followers (not shown); each follower independently deems the meme interesting with probability $\lambda$. Also shown is a later retweet event, which also copies meme $M$. (b) The retweet tree for meme $M$, seeded at time $\tau$. Each retweet by user $A$ of meme $M$ generates a new branch on this tree; each branch can also generate further retweets by followers of $A$, and these subtrees are denoted by squares. (c) Schematic depiction of Eqs. (7) and (16). 
meme $M$ that entered the stream at time $\tau$ (either by innovation or because it was received from a followed user and deemed interesting by $A$ ); see Fig. 3(a).

The likelihood that meme $M$ is retweeted in the future depends on how quickly other tweets enter the stream of user $A$. In fact, meme $M$ can be considered to "occupy" the stream for a time interval $\ell$ stretching from $\tau$ until the time $\tau+\ell$ when the next interesting meme enters the stream of user $A$. New memes enter the stream as a Poisson process at the constant rate ${ }^{1}$

$$
r_{j k}=j \bar{\beta} \lambda+\mu \beta_{j k},
$$

so the occupation time $\ell$ of meme $M$-the time it occupies the stream of user $A$-is an exponentially distributed random variable with density

$$
P_{\text {occ }}(\ell)=r_{j k} \exp \left(-r_{j k} \ell\right) .
$$

We note in passing that the mean occupation time

$$
\langle\ell\rangle=\int_{0}^{\infty} \ell P_{\mathrm{occ}}(\ell) d \ell=\frac{1}{j \bar{\beta} \lambda+\mu \beta_{j k}}
$$

is, for small innovation probabilities $\mu$, inversely proportional to $j$, the number of users followed. Thus, a user who follows many others experiences tweets entering his stream at a higher rate than a lower- $j$ user (compare the streams of users $B$ and $C$ in the schematic of Fig. 1). Consequently, the high- $j$ user is less likely to see (and so to retweet) a given meme than a low- $j$ user. This aspect of the model clearly reflects empirical data, as seen in Fig. 3 of Ref. [51], for example.

To determine the size of trees originating from meme $M$, we consider that trees observed at a time $\Omega \geq \tau$ must be created by the retweeting by user $A$, at some time(s) between $\tau$ and $\Omega$, via looking back in her stream to a time $r$, where $r$ lies between $\tau$ and $\min (\tau+\ell, \Omega)$ (i.e., $r$ lies within the time interval where meme $M$ occupies the stream). Let us consider a time interval of (small) length $d r$, centered at time $r$, and calculate the size of the trees that are seeded by a retweet based on a lookback into this interval, from a time $t$, with $t>r$ (see Fig. 3). In each $d t$ interval centered at time $t$, a tree will be seeded with probability ${ }^{2}$

\footnotetext{
${ }^{1}$ User $A$ follows $j$ users, each of which is assumed to tweet at the average rate $\bar{\beta}=\sum_{j k}(k / z) \beta_{j k} p_{j k}$. Each meme sent by these $j$ users is deemed interesting by $A$ with probability $\lambda$, so the rate at which interesting memes enter the stream of user $A$ is $j \bar{\beta} \lambda$. Moreover, user $A$ innovates at a rate $\mu \beta_{j k}$, which gives the second term of Eq. (2). If either an incoming tweet or an innovation event occurs, a new meme is inserted into the stream of user $A$, and the occupation time of meme $M$ ends.

${ }^{2}$ The factor $(1-\mu) \beta_{j k} d t$ is the probability that a $(j, k)$-class user becomes active in the $d t$ interval and copies rather than innovates; the factor $\Phi(t-r) d r$ is the probability that this user chooses to copy from the $d r$ interval.
}

$$
P_{\text {seed }}=(1-\mu) \beta_{j k} \Phi(t-r) d r d t
$$

and will grow to a tree with size distribution (at observation time $\Omega$ ) generated by ${ }^{3}$

$$
R_{k}(t, \Omega ; x)=x[1-\lambda+\lambda G(t, \Omega ; x)]^{k},
$$

where

$$
G(t, \Omega ; x)=\sum_{j, k} \frac{j}{z} p_{j k} G_{j k}(t, \Omega ; x)
$$

is the PGF for the sizes of trees originating from the successful insertion at time $t$ of a meme (that is deemed interesting) into the stream of a random follower.

To calculate the total size of the tree seeded by copying from the $d r$ interval, we must add the sizes of trees that are copied into all times $t$ with $t>r$. Since each copying event is independent, the total tree size is generated by

$$
J(r ; x)=\prod_{t=r}^{\Omega}\left[1-P_{\text {seed }}+P_{\text {seed }} R_{k}(t, \Omega ; x)\right] .
$$

Taking logarithms of both sides of this equation and expanding to first order in $d t$ gives

$$
\begin{aligned}
\log J= & \sum_{t=r}^{\Omega} \log \left[1-(1-\mu) \beta_{j k} \Phi(t-r) d r d t\right. \\
& \left.\times\left(1-R_{k}(t, \Omega ; x)\right)\right] \\
\approx & -(1-\mu) \beta_{j k} \sum_{t=r}^{\Omega} \Phi(t-r) d r d t\left(1-R_{k}(t, \Omega ; x)\right) \\
& \rightarrow-(1-\mu) \beta_{j k} d r \int_{r}^{\Omega} \Phi(t-r)\left(1-R_{k}(t, \Omega ; x)\right) d t \\
& \text { as } d t \rightarrow 0,
\end{aligned}
$$

so $J(r ; x)$ can be written as

$$
\begin{aligned}
J(r ; x)= & \exp \left[-(1-\mu) \beta_{j k} d r \int_{r}^{\Omega} \Phi(t-r)\right. \\
& \left.\times\left(1-R_{k}(t, \Omega ; x)\right) d t\right]
\end{aligned}
$$

Recall that $J(r ; x)$ is the PGF for trees seeded by copying from time $r$. To obtain the total size of all children trees of meme $M$, we must consider trees seeded at all possible

\footnotetext{
${ }^{3}$ There are $k$ followers of user $A$, each of whom may deem the tweet "uninteresting" with probability $1-\lambda$, or consider it "interesting"-and accept it into their stream-with probability $\lambda$. The factor of $x$ counts the increase in popularity due to the tweet event.
} 
times $r$ from $\tau$ to the time $\min (\tau+\ell, \Omega)$ that marks the end of the occupation of user $A$ 's stream by meme $M$. Each $d r$ time interval again independently generates trees with sizes distributed according to Eq. (10), so the PGF for the total size is found by multiplying together copies of the $J(r ; x)$ function for each $d r$ time interval; thus,

$$
\begin{aligned}
P_{\text {size }}(\ell) & =\prod_{r=\tau}^{\min (\tau+\ell, \Omega)} J(r ; x) \\
& =\exp \left[-(1-\mu) \beta_{j k} \sum_{r=\tau}^{\min (\tau+\ell, \Omega)} d r \int_{r}^{\Omega} \Phi(t-r)\left(1-R_{k}(t, \Omega ; x)\right) d t\right] \\
& \rightarrow \exp \left[-(1-\mu) \beta_{j k} \int_{\tau}^{\min (\tau+\ell, \Omega)} d r \int_{r}^{\Omega} d t \Phi(t-r)\left(1-R_{k}(t, \Omega ; x)\right)\right] \quad \text { as } d r \rightarrow 0 .
\end{aligned}
$$

Combining probabilities, by integrating over all possible occupation times $\ell$, gives

$$
G_{j k}(\tau, \Omega ; x)=\int_{0}^{\infty} P_{\text {occ }}(\ell) P_{\text {size }}(\ell) d \ell,
$$

and combining Eqs. (3), (7), and (11) yields an integral equation for $G$ :

$$
\begin{aligned}
G(\tau, \Omega ; x)= & \sum_{j k} \frac{j}{z} p_{j k} \int_{0}^{\infty} d \ell\left(j \bar{\beta} \lambda+\mu \beta_{j k}\right) \exp \left[-\left(j \bar{\beta} \lambda+\mu \beta_{j k}\right) \ell\right] \\
& \times \exp \left[-(1-\mu) \beta_{j k} \int_{0}^{\min (\tau+\ell, \Omega)} d r \int_{r}^{\Omega} d t \Phi(t-r)\left(1-x[1-\lambda+\lambda G(t, \Omega ; x)]^{k}\right)\right] .
\end{aligned}
$$

Introducing the change of variables $a=\Omega-\tau, \tilde{r}=r-\tau, \tilde{\tau}=\Omega-t$, we rewrite this equation as

$$
\begin{aligned}
G(\Omega-a, \Omega ; x)= & \sum_{j k} \frac{j}{z} p_{j k} \int_{0}^{\infty} d \ell\left(j \bar{\beta} \lambda+\mu \beta_{j k}\right) \exp \left[-\left(j \bar{\beta} \lambda+\mu \beta_{j k}\right) \ell\right] \\
& \times \exp \left[-(1-\mu) \beta_{j k} \int_{0}^{\min (\ell, a)} d \tilde{r} \int_{0}^{a-\tilde{r}} d \tilde{\tau} \Phi(a-\tilde{r}-\tilde{\tau})\left(1-x[1-\lambda+\lambda G(\Omega-\tilde{\tau}, \Omega ; x)]^{k}\right)\right] .
\end{aligned}
$$

Note that the only appearance of the observation time $\Omega$ in this equation is in the first two arguments of the $G$ function; this reflects the fact that the popularity of memes in this model depends only on their age $a$ (unlike cumulative-advantage models, which exhibit a dependence also on the global time because early-born items have an "early-mover" advantage [52]). We therefore compress the notation by defining $G$ in terms only of the age $a$ of the memes: $G(\Omega-\tau ; x):=G(\tau, \Omega ; x)$, and $G(a ; x)$ solves the integral equation

$$
\begin{aligned}
G(a ; x)= & \sum_{j k} \frac{j}{z} p_{j k} \int_{0}^{\infty} d \ell\left(j \bar{\beta} \lambda+\mu \beta_{j k}\right) \exp \left[-\left(j \bar{\beta} \lambda+\mu \beta_{j k}\right) \ell\right] \\
& \times \exp \left[-(1-\mu) \beta_{j k} \int_{0}^{\min (\ell, a)} d \tilde{r} \int_{0}^{a-\tilde{r}} d \tilde{\tau} \Phi(a-\tilde{r}-\tilde{\tau})\left(1-x[1-\lambda+\lambda G(\tilde{\tau} ; x)]^{k}\right)\right],
\end{aligned}
$$

with initial condition $G(0 ; x)=1$.

The popularity of a meme, as observed at time $\Omega$, that is seeded by a single tweet (e.g., by an innovation) at time $\tau$ may be calculated in a similar way to the derivation of Eq. (15); the generating function is of the form

$$
H(\tau, \Omega ; x)=\sum_{j, k} \beta_{j k} p_{j k} R_{k}(\tau, \Omega ; x) G_{j k}(\tau, \Omega ; x),
$$

where $\beta_{j k} p_{j k}$ represents the probability that the seed tweet originates from a $(j, k)$-class user, $R_{k}$ is the PGF for the trees generated from the followers of the user, and $G_{j k}$ is the PGF for the size of the retweet tree of the meme [see Fig. 3(c)]. Introducing the age $a$ of the meme as before and defining $q_{n}(a)$ as the probability that an age- $a$ meme has popularity $n$, we have the PGF defined in Eq. (1), which is given by 


$$
\begin{aligned}
H(a ; x)= & \sum_{j k} \beta_{j k} p_{j k} x[1-\lambda+\lambda G(a ; x)]^{k} \int_{0}^{\infty} d \ell\left(j \bar{\beta} \lambda+\mu \beta_{j k}\right) \exp \left[-\left(j \bar{\beta} \lambda+\mu \beta_{j k}\right) \ell\right] \\
& \times \exp \left[-(1-\mu) \beta_{j k} \int_{0}^{\min (\ell, a)} d \tilde{r} \int_{0}^{a-\tilde{r}} d \tilde{\tau} \Phi(a-\tilde{r}-\tilde{\tau})\left(1-x[1-\lambda+\lambda G(\tilde{\tau} ; x)]^{k}\right)\right]
\end{aligned}
$$

the initial condition is $H(0 ; x)=x$ [i.e., all memes have initial popularity $\left.1: q_{n}(0)=\delta_{n, 1}\right]$.

\section{B. Distribution of response times}

It is worth noting that all agents in the model have constant activity rates, so the actions of each individual agent constitute a Poisson process. A Poisson process is characterized by an exponential distribution of interevent times, where each event corresponds to an innovation or a retweeting action. This assumption is contrary to studies such as Refs. [29,30,33-35,53-59], where heavy-tailed distributions of interevent times are examined. Despite this, in our model the memory-time distribution $\Phi\left(t_{m}\right)$ directly influences the waiting times (or "response times") between the receipt of a specific meme and the retweeting of it. Indeed, if $\Phi\left(t_{m}\right)$ is a heavy-tailed distribution, then a meme received by a given user at time $\tau$ will be retweeted by that user at a time $t$ (with $t \gg \tau$ ) with probability proportional to $\Phi(t-\tau)$ (the exact relation depends on how long the meme occupies the stream of the user). Therefore, a heavy-tailed memory distribution gives rise to a heavy-tailed waiting-time distribution for individual memes, despite the fact that the activity of each individual user is described by a Poisson process (cf. the heavy-tailed waiting-time distributions found in empirical studies of email correspondence [29,30]). It is clearly important to distinguish between the distributions of interevent times (for actions of users) and of the waiting times experienced by individual memes; the model assumes each user has exponentially distributed interevent times, but it can nevertheless produce heavy-tailed distributions of waiting times for memes to be retweeted.

In particular, if the memory-time distribution $\Phi\left(t_{m}\right)$ is a $\operatorname{Gamma}\left(k_{G}, \theta\right)$ distribution [35] as used in Secs. V and VI, i.e., $\Phi\left(t_{m}\right)=\left\{1 /\left[\Gamma\left(k_{G}\right) \theta^{k_{G}}\right]\right\} t_{m}^{k_{G}-1} \exp \left(-t_{m} / \theta\right)$, then $\Phi\left(t_{m}\right)$ is approximately a power law for memory times $t_{m}$ with $t_{m} \ll \theta$, with an exponential cutoff at larger times. The corresponding waiting-time distribution shows a similar scaling in this range, like the slow decay noted in empirical response times for Twitter users (e.g., in Fig. 5 of Ref. [51]). In Sec. VII we consider how the model could be extended to incorporate bursty (non-Poisson) user activity.

\section{ANALYSIS}

\section{A. Criticality of the branching process}

A branching process may be classified by the expected (mean) number $\xi$ of "children" of each "parent": If this number (called the "branching number") is less than 1, the process is subcritical, and if $\xi$ is greater than 1, the process is supercritical. Critical branching processes, with an average of exactly one child per parent, give rise to power-law distributions of tree sizes and of durations of growth cascades, and have been used to examine selforganized criticality in sandpile models on networks $[47,50]$. Here, we demonstrate that the general process derived in Sec. III is a critical branching process in the limit of vanishing innovation $\mu \rightarrow 0$.

We identify the parent in the process as a meme that was accepted into the stream (i.e., deemed interesting) of a $(j, k)$-class user at time $\tau$ (see, for example, meme $M$ in the stream of user $A$, as shown in Fig. 3). The children of this meme are the retweets of it that are accepted into the streams of the followers of $A$ at any time $t>\tau$. The PGF for the number of children of meme $M$ is derived by following the same steps as in Sec. III but replacing $R_{k}$ by $(1-\lambda+\lambda x)^{k}$; each power of $x$ then counts a successful insertion of meme $M$ into the stream of one of the $k$ followers of $A$. The resulting PGF, for a meme of age $a$, is [cf. Eq. (13)]

$$
\begin{aligned}
K_{j k}(a ; x)= & \int_{0}^{\infty} d \ell P_{\mathrm{occ}}(\ell) \exp \left[-(1-\mu) \beta_{j k} \int_{0}^{\min (\ell, a)} d \tilde{r}\right. \\
& \left.\times \int_{0}^{a-\tilde{r}} d \tilde{\tau} \Phi(a-\tilde{r}-\tilde{\tau})\left(1-[1-\lambda+\lambda x]^{k}\right)\right] \\
= & \int_{0}^{\infty} d \ell P_{\mathrm{occ}}(\ell) \exp \left[-(1-\mu) \beta_{j k}\right. \\
& \left.\times\left(1-[1-\lambda+\lambda x]^{k}\right) \int_{0}^{\min (\ell, a)} C(a-\tilde{r}) d \tilde{r}\right],
\end{aligned}
$$

where $C(t)=\int_{0}^{t} \Phi\left(t_{m}\right) d t_{m}$ is the cumulative distribution function $(\mathrm{CDF})$ for memory times. The expected (mean) number of children for a meme in the $(j, k)$-class stream is determined from the PGF in the usual way [45], by differentiating with respect to $x$ and evaluating at $x=1$; thus,

$$
\xi_{j k}=\left.\frac{\partial K_{j k}}{\partial x}\right|_{x=1}
$$

In the limit of large ages, $a \rightarrow \infty$, we use the fact that $C(\infty)=1$ to obtain 


$$
\begin{aligned}
\xi_{j k} & \sim(1-\mu) \beta_{j k} \lambda k \int_{0}^{\infty} \ell P_{\mathrm{occ}}(\ell) d \ell \quad \text { as } a \rightarrow \infty \\
& =\frac{(1-\mu) \beta_{j k} \lambda k}{j \bar{\beta} \lambda+\mu \beta_{j k}} .
\end{aligned}
$$

Averaging over all $(j, k)$ classes, the effective branching number $\xi$ of the process is the expected number of children of a meme that is accepted into the stream of a random follower:

$$
\begin{aligned}
\xi & =\sum_{j, k} \frac{j}{z} p_{j k} \xi_{j k} \\
& \rightarrow \sum_{j, k} \frac{j}{z} p_{j k} \frac{\beta_{j k} \lambda k}{j \bar{\beta} \lambda}=1 \quad \text { as } \mu \rightarrow 0
\end{aligned}
$$

(recall that $\bar{\beta} \equiv \sum_{j, k}(k / z) \beta_{j k} p_{j k}$ ).

Thus, we have shown that the branching process underlying the model is critical when $\mu=0$. The occupation time of a meme in a users' stream is due to the competition between neutral-fitness memes for the limited resource of user attention; this competition ensures that the mean number of successful retweets (children) generated during the finite occupation time of the meme is precisely one, and thus, it induces the power-law distributions of cascade sizes that are characteristic of critical branching processes $[47,50]$.

It is worth noting that the result of Eq. (21) can also be derived in a more heuristic fashion, which enables us to discuss possible generalizations of the model in Sec. VII. As above, we want to calculate $\xi_{j k}$, the expected number of children of a parent meme $M$ that has been accepted into the stream of a $(j, k)$-class user, called user $A$. We consider a (long) time window of duration $W$ units. During this time window, a total of approximately $\left(j \bar{\beta} \lambda+\mu \beta_{j k}\right) W$ tweets have been accepted into the stream of user $A$ [see footnote 1 and Eq. (4)]. When user $A$ decides to retweet during the time window, one of these memes is chosen for retweeting. If the times chosen by the user are uniformly distributed over the window, then the probability that the chosen meme is meme $M$ is

$$
\begin{aligned}
P_{\text {chosen }} & =\frac{1}{\text { number of memes in stream }} \\
& =\frac{1}{\left(j \bar{\beta} \lambda+\mu \beta_{j k}\right) W} .
\end{aligned}
$$

Alternatively, this result can be calculated by noting that the average time that a single meme occupies the stream is given by $\langle\ell\rangle$ in Eq. (4), so the expected fraction of the total time that meme $M$ occupies the stream of user $A$ over the window of length $W$ is $\langle\ell\rangle / W=P_{\text {chosen }}$.

Recalling that the activity rate of user $A$ is $\beta_{j k}$, the expected number of retweets by this user during the time window is

$$
N_{\text {retweets }}=(1-\mu) \beta_{j k} W .
$$

Each retweet is broadcast to the $k$ followers of $A$, each of whom finds the retweet interesting with probability $\lambda$, so the expected number of children (memes deemed interesting by followers) per retweet is $\lambda k$. The expected number of children of the parent meme $M$ over the time window is therefore

$$
\begin{aligned}
\xi_{j k}= & (\text { number of retweets by } A) \\
& \times(\text { probability meme } M \text { is chosen }) \\
& \times(\text { children per retweet }) \\
= & N_{\text {retweets }} P_{\text {chosen }} \lambda k
\end{aligned}
$$

which recovers Eq. (20). The expected number $\xi$ of children of a meme that is accepted into the stream of a random follower is then calculated as in Eq. (21), giving $\xi \rightarrow 1$ in the $\mu \rightarrow 0$ limit.

\section{B. An explicit expression for $q_{1}(a)$}

The value $q_{1}(a)$ is the probability that a meme, once created via an innovation event, is not retweeted by the time it reaches age $a$ : Recall that the popularity $n$ of a meme is set to 1 when it is first tweeted (i.e., at birth); subsequent retweets (if any) increase the value of $n$ above 1 . The probability $q_{1}(a)$ may be calculated explicitly using Eq. (17):

$$
\begin{aligned}
q_{1}(a)= & \lim _{x \rightarrow 0} \frac{H(a ; x)}{x}, \\
= & \sum_{j, k} \beta_{j k} p_{j k}[1-\lambda+\lambda G(a ; 0)]^{k} \int_{0}^{\infty} d \ell P_{\mathrm{occ}}(\ell) \\
& \times \exp \left[-(1-\mu) \beta_{j k} \int_{0}^{\min (\ell, a)} C(a-\tilde{r}) d \tilde{r}\right],
\end{aligned}
$$

with $G(a ; 0)$ given, from Eq. (15), by

$$
\begin{aligned}
G(a ; 0)= & \sum_{j, k} \frac{j}{z} p_{j k} \int_{0}^{\infty} d \ell P_{\mathrm{occ}}(\ell) \\
& \times \exp \left[-(1-\mu) \beta_{j k} \int_{0}^{\min (\ell, a)} C(a-\tilde{r}) d \tilde{r}\right] .
\end{aligned}
$$

If we consider the large-age limit, $a \rightarrow \infty$, then we can approximate the integral of the cumulative distribution function for memory times as

$$
\int_{0}^{\min (\ell, a)} C(a-\tilde{r}) d \tilde{r} \approx \ell C(a)
$$

and the integral over $\ell$ can be calculated to give the large- $a$ approximation 


$$
\begin{aligned}
q_{1}(a) \sim & \sum_{j k} \beta_{j k} p_{j k} \frac{j \bar{\beta} \lambda+\mu \beta_{j k}}{j \bar{\beta} \lambda+\mu \beta_{j k}+(1-\mu) \beta_{j k} C(a)} \\
& \times[1-\lambda+\lambda G(a ; 0)]^{k}
\end{aligned}
$$

with

$$
G(a ; 0) \sim \sum_{j k} \frac{j}{z} p_{j k} \frac{j \bar{\beta} \lambda+\mu \beta_{j k}}{j \bar{\beta} \lambda+\mu \beta_{j k}+(1-\mu) \beta_{j k} C(a)} .
$$

In the simplified case $p_{j k}=\delta_{j, z} p_{k}$ and $\beta_{j k} \equiv 1$, Eqs. (28) and (29) reduce to

$$
\begin{aligned}
q_{1}(a) \sim & \frac{\lambda z+\mu}{\lambda z+\mu+(1-\mu) C(a)} \sum_{k=0}^{\infty} p_{k} \\
& \times\left[1-\lambda+\lambda \frac{\lambda z+\mu}{\lambda z+\mu+(1-\mu) C(a)}\right]^{k} .
\end{aligned}
$$

The $a=\infty$ limit of $q_{1}(a)$ gives the fraction of memes that are never retweeted and thus have popularity $n=1$ forever. The value of $q_{1}(\infty)$ is obtained from Eqs. (28) and (29) by setting $C(a)$ to its $a \rightarrow \infty$ limit of 1 . The approach of $q_{1}(a)$ towards the value $q_{1}(\infty)$ depends, through the $\mathrm{CDF} C(a)$, on the tail of the memory-time distribution $\Phi$. If the distribution $\Phi$ is heavy tailed, there is a non-negligible probability that a meme may be retweeted even if a very long time has elapsed since its birth.

\section{Mean popularity}

The age dependence of the mean popularity (i.e., the expected number of tweets or retweets for a meme of age $a$ ) is given by

$$
m(a)=\sum_{n=1}^{\infty} n q_{n}(a)=\left.\frac{\partial H(a ; x)}{\partial x}\right|_{x=1} .
$$

Differentiating Eq. (17) and setting $x=1$ yields an integral equation for $m(a)$ :

$$
\begin{aligned}
m(a)= & \sum_{j k} \beta_{j k} p_{j k}\left\{1+\lambda k m_{G}(a)+(1-\mu) \beta_{j k}\right. \\
& \times \int_{0}^{\infty} d \ell\left(j \bar{\beta} \lambda+\mu \beta_{j k}\right) \exp \left[-\left(j \bar{\beta} \lambda+\mu \beta_{j k}\right) \ell\right] \\
& \left.\times \int_{0}^{\min (\ell, a)} d \tilde{r} \int_{0}^{a-\tilde{r}} d \tilde{\tau} \Phi(a-\tilde{r}-\tilde{\tau})\left[1+\lambda k m_{G}(\tilde{\tau})\right]\right\},
\end{aligned}
$$

where $m_{G}(a)$, defined by $m_{G}(a)=\left.\{\partial G(a ; x) / \partial x\}\right|_{x=1}$, is the solution of the integral equation found by differentiating Eq. (15):

$$
\begin{aligned}
m_{G}(a)= & \sum_{j k} \frac{j}{z} p_{j k} \int_{0}^{\infty} d \ell\left(j \bar{\beta} \lambda+\mu \beta_{j k}\right) \\
& \times \exp \left[-\left(j \bar{\beta} \lambda+\mu \beta_{j k}\right) \ell\right](1-\mu) \beta_{j k} \int_{0}^{\min (\ell, a)} \\
& \times d \tilde{r} \int_{0}^{a-\tilde{r}} d \tilde{\tau} \Phi(a-\tilde{r}-\tilde{\tau})\left[1+\lambda k m_{G}(\tilde{\tau})\right] .
\end{aligned}
$$

The order of the time integrals may be swapped using the identity

$$
\int_{0}^{\infty} d \ell \int_{0}^{\min (\ell, a)} d \tilde{r}=\int_{0}^{a} d \tilde{r} \int_{\tilde{r}}^{\infty} d \ell,
$$

and the resulting $\ell$ integral can be performed explicitly:

$$
\int_{\tilde{r}}^{\infty}\left(j \bar{\beta} \lambda+\mu \beta_{j k}\right) e^{-\left(j \bar{\beta} \lambda+\mu \beta_{j k}\right) \ell} d \ell=e^{-\left(j \bar{\beta} \lambda+\mu \beta_{j k}\right) \tilde{r}}
$$

As a result, the expressions (32) and (33) can be written as double convolution integrals. Taking Laplace transforms, Eq. (32) then becomes

$$
\begin{aligned}
\hat{m}(s)= & \frac{1}{s}+z \bar{\beta} \lambda \hat{m}_{G}(s)+(1-\mu) \hat{\Phi}(s) \sum_{j, k} \beta_{j k}^{2} p_{j k} \\
& \times \frac{\frac{1}{s}+\lambda k \hat{m}_{G}(s)}{j \bar{\beta} \lambda+\mu \beta_{j k}+s},
\end{aligned}
$$

where hats denote Laplace transforms, e.g.,

$$
\hat{\Phi}(s) \equiv \int_{0}^{\infty} e^{-s t} \Phi(t) d t,
$$

and with $\hat{m}_{G}(s)$ given explicitly from the Laplace transform of Eq. (33):

$$
\hat{m}_{G}(s)=\frac{(1-\mu) \hat{\Phi}(s) \sum_{j, k} \frac{j}{z} p_{j k} \frac{\beta_{j k}}{s \bar{\beta} \lambda+\mu \beta_{j k}+s}}{s\left[1-(1-\mu) \lambda \hat{\Phi}(s) \sum_{j, k} \frac{j}{z} p_{j k} \frac{k \beta_{j k}}{j \bar{\beta} \lambda+\mu \beta_{j k}+s}\right]} .
$$

If we specialize now to the simplified case where $\beta_{j k} \equiv 1$ for all $(j, k)$ classes, and $p_{j k}=\delta_{j, z} p_{k}$, we obtain the simpler expression

$$
\hat{m}_{G}(s)=\frac{(1-\mu) \hat{\Phi}(s) \frac{1}{\lambda z+\mu+s}}{s\left[1-(1-\mu) \hat{\Phi}(s) \frac{\lambda z}{\lambda z+\mu+s}\right]} .
$$

Substituting for $\hat{m}_{G}$ into the simplified version of Eq. (36) yields

$$
\hat{m}(s)=\frac{1}{s}+\frac{1-\mu}{s} \frac{(\lambda z+1) \hat{\Phi}(s)}{\lambda z+\mu+s-(1-\mu) \lambda z \hat{\Phi}(s)} .
$$

Note that, unlike the expression for $q_{1}$ in Eq. (30), the mean popularity depends on the out-degree distribution $p_{k}$ only through the mean degree $z$, implying that the mean 
popularity is independent of the finer details of the network structure.

To consider the large-age asymptotics of $m(a)$ from Eq. (40), we use results from renewal theory [35,60]. If the Malthusian parameter $\alpha$ exists, where $\alpha$ is defined as the solution of the equation

$$
\frac{(1-\mu) \lambda z \hat{\Phi}(\alpha)}{\lambda z+\mu+\alpha}=1
$$

then the large-age, small- $\mu$ asymptotic behavior of $m(a)$ can be written as (Theorem IV.4.2 of Ref. [60])

$$
m(a) \sim \frac{1}{\mu}-\frac{1}{\mu} e^{-\frac{\mu(1 \lambda+1)}{1+T \lambda z} a} \quad \text { as } a \rightarrow \infty, \mu \rightarrow 0 .
$$

Here, we have used the fact that near criticality (i.e., as $\mu \rightarrow 0$ ), the Malthusian parameter $\alpha$ is determined by Eq. (41) to be $\alpha=-[\mu(\lambda z+1)] /(1+T \lambda z)+O\left(\mu^{2}\right)$, where $T=\int_{0}^{\infty} t_{m} \Phi\left(t_{m}\right) d t_{m}$ is the mean memory time. ${ }^{4}$ Setting $a=\infty$ in Eq. (42), we obtain the steady-state value of the mean popularity, $m(\infty)=1 / \mu$. Although Eq. (42) is a large- $a$ asymptotic result, we may expand the exponential term about $a=0$, provided that the argument of the exponential remains small; this is valid for ages $a$ that obey the constraint $a \ll(1+T \lambda z) /[\mu(\lambda z+1)]$. Taking the $\mu \rightarrow 0$ limit of Eq. (42) shows that the function $m(a)$ grows linearly with $a$ for ages in this range:

$$
m(a) \sim \frac{\lambda z+1}{1+T \lambda z} a .
$$

The preceding analysis all assumes that the seed node (i.e., the user who first tweets the meme of interest) is chosen at random from all the network users, with probability weighted by the user activity rate. It is straightforward to repeat the steps of the calculations for the case where the seed node is known to have $k$ followers and thus to investigate the importance of the connectivity of the seed node. Restricting our attention to the simplified case as above and taking the infinite-age limit, we find that the expected popularity for a meme that is initiated by a seed node of out-degree $k$ is

\footnotetext{
${ }^{4}$ Note that the Malthusian parameter exists for all the memorytime distributions considered in this paper (exponential and gamma distributions). However, if $\Phi$ is a subexponential distribution [60] (such as the log-normal distribution [61]), then the large- $a$ asymptotics of the mean popularity are related to the memory-time CDF by

$$
m(a) \sim \frac{1}{\mu}-\frac{(1-\mu)(\lambda z+\mu)}{\mu^{2}(\lambda z+1)}(1-C(a))
$$

instead of Eq. (42).
}

$$
m_{k}(\infty)=\frac{\lambda z+1}{\lambda z+\mu}\left(1+\frac{\lambda(1-\mu)}{\mu(\lambda z+1)} k\right)
$$

Note the linear dependence of this expression on the number of followers $k$ of the seed node: Memes tweeted by users with a large number of followers are likely to become more popular than memes seeded by less influential nodes. This feature of the model matches well to the observed dependence of the size of information cascades on the connectivity of the initial seed (e.g., Fig. 2 of Ref. [62]). Of course, the earlier results for randomly chosen seeds are recovered by averaging over all possible seed nodes: $m(\infty)=\sum_{k} p_{k} m_{k}(\infty)=1 / \mu$.

\section{Infinite-age limit of popularity distribution}

In the infinite-age (steady-state) limit $a \rightarrow \infty$, we assume $G(a ; x) \rightarrow G_{\infty}(x)$, independent of $a$, and use the fact that $\int_{0}^{\infty} \Phi(t) d t=1$ in Eq. (15) to obtain

$$
\begin{aligned}
G_{\infty}(x)= & \sum_{j k} \frac{j}{z} p_{j k} \int_{0}^{\infty} d \ell\left(j \bar{\beta} \lambda+\mu \beta_{j k}\right) \\
& \times \exp \left[-\left(j \bar{\beta} \lambda+\mu \beta_{j k}\right) \ell\right] \\
& \times \exp \left[-(1-\mu) \beta_{j k} \ell\left(1-x\left[1-\lambda+\lambda G_{\infty}(x)\right]^{k}\right)\right] .
\end{aligned}
$$

Calculating the $\ell$ integral then gives the equation satisfied by $G_{\infty}(x)$ :

$G_{\infty}(x)=\sum_{j k} \frac{j}{z} p_{j k} \frac{j \bar{\beta} \lambda+\mu \beta_{j k}}{j \bar{\beta} \lambda+\beta_{j k}-(1-\mu) \beta_{j k} x\left[1-\lambda+\lambda G_{\infty}(x)\right]^{k}}$.

Similarly, the infinite-age limit for $H$ is given in terms of $G_{\infty}$ by

$H_{\infty}(x)=\sum_{j k} \beta_{j k} p_{j k} \frac{\left(j \bar{\beta} \lambda+\mu \beta_{j k}\right) x\left[1-\lambda+\lambda G_{\infty}(x)\right]^{k}}{j \bar{\beta} \lambda+\beta_{j k}-(1-\mu) \beta_{j k} x\left[1-\lambda+\lambda G_{\infty}(x)\right]^{k}}$.

Note that these steady-state equations are independent of the memory distribution function $\Phi$. Accordingly, the asymptotic analysis approach used in Ref. [37] to obtain the large- $n$ behavior of the popularity distribution $q_{n}(\infty)$ may also be applied here; this is based on writing $x=$ $1-w$ and $G_{\infty}=1-\phi(w)$ and analyzing the small-w, small- $\phi$ asymptotics of Eqs. (46) and (47). We refer to Ref. [37] for details, and here, we summarize the main results for the simplified case $\beta_{j k} \equiv 1, p_{j k}=\delta_{j, z} p_{k}$.

(i) Case 1: $p_{k}$ has a finite second moment.

The large- $n$ scaling of the popularity distribution is given by a power-law with exponential cutoff: 


$$
q_{n}(\infty) \sim A n^{-\frac{3}{2}} e^{-(n / k)} \quad \text { as } n \rightarrow \infty,
$$

where the prefactor $A$ is ${ }^{5}$

$$
A=\frac{z(\lambda z+1)}{\lambda z+\mu}\left[2 \pi\left(\frac{\left\langle k^{2}\right\rangle(2+\lambda z-\mu)}{\lambda z+\mu}-z\right)\right]^{-\frac{1}{2}}
$$

and the cutoff $\kappa$ is

$$
\kappa=\frac{2 \lambda^{2}(1-\mu)^{2}}{\mu^{2}(\lambda z+1)^{2}}\left[\frac{\left\langle k^{2}\right\rangle(2+\lambda z-\mu)}{\lambda z+\mu}-z\right] .
$$

Note that $\kappa$ is proportional to $1 / \mu^{2}$ for small $\mu$, so in the limit of vanishing innovation probability, the exponential cutoff tends to infinity and the power-law part of the popularity distribution extends to all $n$.

(ii) Case 2: $p_{k} \sim D k^{-\gamma}$ as $k \rightarrow \infty$, with $\gamma$ between 2 and 3. Immediately taking the $\mu \rightarrow 0$ limit, we find in this case that the popularity distribution has a power-law form with exponent $\gamma /(\gamma-1)$ lying between $3 / 2$ and 2 [47,48]:

$$
q_{n}(\infty) \sim B n^{-[\gamma /(\gamma-1)]} \quad \text { as } n \rightarrow \infty
$$

with prefactor $B$ given by

$$
\begin{aligned}
B= & -(\lambda z+1) \frac{(D \Gamma(1-\gamma))^{-[1 /(\gamma-1)]}}{\lambda \Gamma\left(\frac{1}{1-\gamma}\right)} \\
& \times\left[(\lambda z)^{2} \sum_{n=1}^{\infty} \frac{n^{\gamma-1}}{(\lambda z+1)^{n+1}}\right]^{-[1 /(\gamma-1)]},
\end{aligned}
$$

where $\Gamma$ is the gamma function.

\section{E. Large- $a$, large- $n$ asymptotics of popularity distribution}

In Appendix A we consider how the popularity distribution $q_{n}(a)$ behaves for large, but finite, ages, focusing on the case $\beta_{j k} \equiv 1, p_{j k}=\delta_{j, z} p_{k}$ for simplicity. The result of the asymptotic analysis is an expression for the Laplace transform of the PGF $H(a ; x)$ that is valid in the $a \rightarrow \infty$ limit [see Eqs. (A11) and (A12) for the cases of out-degree distributions $p_{k}$ that have second moments $\left\langle k^{2}\right\rangle$ that are, respectively, infinite or finite].

\section{RESULTS: NUMERICAL SIMULATION}

To confirm the accuracy of the branching-process approximation and to explore the interactions of the

\footnotetext{
${ }^{5}$ The values of $A, \kappa$, and $B$ reported here are not identical to those reported in Ref. [37]; this is because of an approximation made in the analysis of Ref. [37] that is not required here [see Eq. (S6) of Ref. [37]]. However, the differences are of order $1 /(\lambda z)$ and thus are negligible in the case $\lambda z \gg 1$ that is considered in Ref. [37].
}

network structure and the memory-time distribution, here we compare numerical simulations of the model with the theoretical predictions of Sec. IV. We generate configuration-model directed networks with prescribed out-degree distribution $p_{k}$. Each one of $N$ users (nodes) is assigned a random number $k$ (drawn from the distribution $p_{k}$ ) of out links (links to followers). The identities of the $k$ followers are chosen uniformly at random from the set of all users; in the $N \rightarrow \infty$ limit, this gives a Poisson in-degree distribution $p_{j}$ which, for sufficiently large $z$, gives similar results to using the in-degree distribution $p_{j}=\delta_{j, z}$, i.e., assuming every user follows exactly $z$ others [37]. Each user has the same activity rate, so $\beta_{j k} \equiv 1$.

Figure 4(a) shows the fraction of memes that have popularity greater than or equal to $n$, at age $a$. Black symbols are the results of numerical simulations; the colored curves are determined from the large- $a$, large- $n$, $\mu=0$ asymptotics of Eq. (A11), using the Laplace transform inversion described in Appendix B. The main figure in panel Fig. 4(a) shows results for networks with the scalefree out-degree distribution $p_{k} \sim D k^{-\gamma}$ for $k \geq 4$ and exponent $\gamma=2.5$ (with $p_{k}=0$ for $k<4$ ); the inset shows the results for networks with a Poisson out-degree distribution with mean degree $z=11$ matching that of the scale-free networks. The memory-time distribution is $\Phi=\operatorname{Gamma}\left(k_{G}, \theta\right)$, with $k_{G}=0.1, \theta=50$ for the scalefree case and $k_{G}=0.1, \theta=5$ for the Poisson case; the mean memory time for this distribution is $T=k_{G} \theta$.

Panels (b) and (c) of Fig. 4 show results for various memory-time distributions $\Phi$ on networks with the same scale-free out-degree distribution as used in panel (a), and panels (d) and (e) show the corresponding results for the Poisson network. Panels (b) and (d) show the fraction $q_{1}(a)$ of memes that have not been retweeted by age $a$, along with the large- $a$ asymptotics of Eq. (30). The age dependence of $q_{1}(a)$ is qualitatively similar in panels (b) and (d): Note in both panels that the cases with longer mean memory time $T=5$ (dashed curves) approach their $a \rightarrow \infty$ limit more slowly than the $T=1$ cases (solid curves). However, the limiting value of $q_{1}(a)$ as $a \rightarrow \infty$ is different in the two panels, reflecting the effect of the network structure (out-degree distribution). Using Eq. (30) [with $C(\infty)=1$ ] we obtain $q_{1}(\infty)=0.50$ for the scale-free network with $\lambda=1$, whereas $q_{1}(\infty)=0.37$ for the Poisson network.

The mean popularity $m(a)$ of age- $a$ memes is shown in panels (c) and (e) for the scale-free and Poisson networks, respectively, and for the same memory-time distributions as used in panels (b) and (d). In contrast to the results for $q_{1}(a)$, we see that the finer details of the network structure have no effect on the $m(a)$ curves: Panels (c) and (e) are identical because Eq. (40) depends on $p_{k}$ only through the mean degree $z$, which is identical for both networks. The mean memory time $T$ determines the rate of linear growth of $m(a)$ at intermediate ages [see Eq. (43)], while at early 

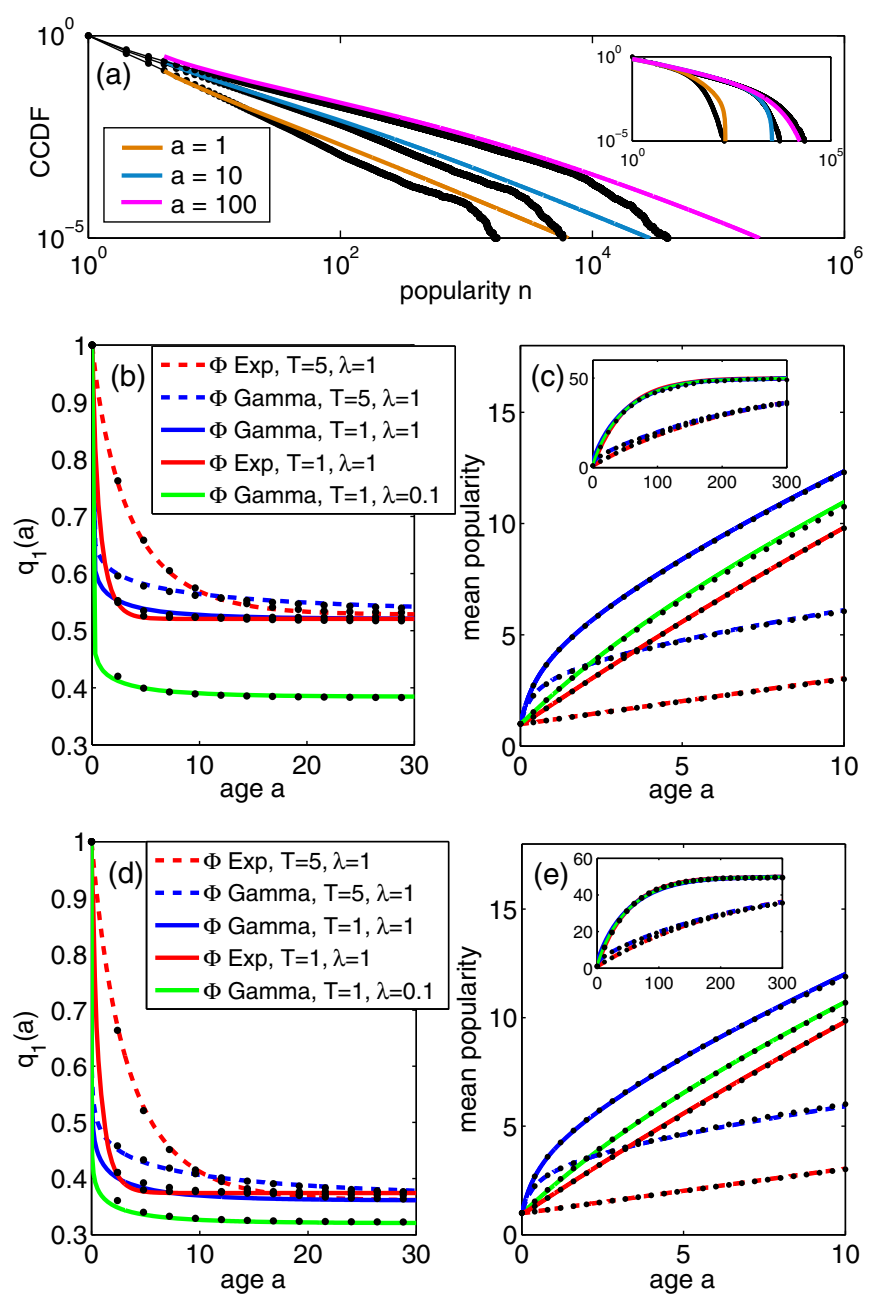

FIG. 4. Numerical simulations of the model, compared with analytical results. (a) Complementary cumulative distribution functions (CCDFs) for meme popularity at age $a$; numerical simulation results (black) on a network with scale-free out-degree distribution ( $p_{k} \propto k^{-\gamma}$ for $k \geq 4$ with $\gamma=2.5$, mean degree $z=11$, $N=10^{5}$ nodes), compared with asymptotic model result Eq. (A11) (colored curves). The memory-time distribution is $\Phi=$ $\operatorname{Gamma}\left(k_{G}, \theta\right)$ with $k_{G}=0.1$ and $\theta=50$, so the mean memory time is $T=k_{G} \theta=5$. Inset: As in the main figure but for Poisson out-degree distribution $p_{k}(z=11)$ and gamma memory-time distribution with $k_{G}=0.1$ and $\theta=0.5$. (b) Fraction $q_{1}(a)$ of memes that are not retweeted by age $a$, on the scale-free network of (a) and for various memory-time distributions $\Phi\left(t_{m}\right)$ [red = exponential with mean $T$; blue $/$ green $=\operatorname{Gamma}(0.1,10 T)$ ], using Eq. (30). Dashed lines show the $T=5$ cases; solid lines represent $T=1$. (c) Mean popularity of memes of age $a$, for the same cases as in (b), and compared with Eq. (40) (using the numerical Laplace transform inversion described in Appendix B); the inset shows the large- $a$ behavior. All panels have $\mu=0.02$ and (except for green curves) $\lambda=1$. (d,e) Same as panels (b) and (c) but for a network with Poisson out-degree distribution (mean degree $z=11$ ), with $\mu=$ 0.02 and (except for green curves) $\lambda=1$.

ages, the gamma memory-time distribution $\Phi\left(t_{m}\right)$ (which has significant probability mass at low values of $t_{m}$ ) gives a faster-than-linear growth of $m(a)$ that is not present for the exponentially distributed memory times. The large-age asymptotics are shown in the insets; as discussed in Sec. IV C, we find $m(a) \rightarrow 1 / \mu$ as $a \rightarrow \infty$. As we show in Sec. VI below, the $m(a)$ curves can be fitted to empirical data on the popularity of Twitter hashtags; note also that the qualitative features identified here (nonlinear early growth; linear intermediate-time growth, saturation at later times) have also been observed in several other measures of information spread on social networks, such as views of YouTube videos [63] and the installation of Facebook apps [5].

\section{RESULTS: TWITTER HASHTAGS DATA}

\section{A. Data and model inputs}

To test the ability of the model to fit real-world data, we use a 1-year data set comprised of the popularities of $1.4 \times$ $10^{5}$ hashtags related to the $201115 \mathrm{M}$ protest movement in Spain, which were tracked over the 1-year period from March 2011 to March 2012 [64,65]. We use all hashtags for which we have at least 200 days of data; each curve in Fig. 6(a) shows the popularity distribution for all hashtags that have the same age (to the nearest day).

The out-degree distribution $p_{k}$ of the Twitter network is an important input to the model. We determine the empirical distribution by randomly selecting $8.2 \times 10^{5}$ Twitter user IDs and recording the number of followers $k$ of each user. The measured mean number of followers is $z=703$, but the distribution $p_{k}$ is heavy tailed. The CCDF of the $k$ values is shown in Fig. 5, along with the line $D /(\gamma-1) k^{1-\gamma}$, with $D=240$ and $\gamma=2.13$, which corresponds to an out-degree distribution with the tail scaling as $p_{k} \sim D k^{-\gamma}$ as $k \rightarrow \infty$ [66].

The model parameter $\lambda$ and the memory-time distribution $\Phi\left(t_{m}\right)$ cannot be directly estimated from the data because in cases where users receive multiple copies of the same meme (hashtag) prior to retweeting it, it is impossible to tell which of the received memes "caused" the retweet. Therefore, we instead use the analytical results of the model [Eqs. (40) and (A11)] to find parameter values that fit the model to the statistical characteristics of the data. Guided by the fasterthan-linear growth of the mean popularity at early ages $a$ [Fig. 6(c)] and the results of Sec. V, we assume that the memory-time distribution $\Phi$ is a $\operatorname{Gamma}\left(k_{G}, \theta\right)$ distribution, and we fit the parameters $k_{G}$ and $\theta$, as well as the model parameters $\mu$ and $\lambda$, to give the results in Fig. 6(c). Note that a delta-function memory-time distribution, as used in the toy model of Ref. [37], leads to a purely linear dependence $m(a) \propto a$, and so we cannot fit to the early-time growth of the observed mean popularity.

The data do, however, provide an upper bound on the value of the innovation probability $\mu$. Recall that $\mu$ is defined as the probability that a tweeted meme (hashtag) is an innovation, i.e., that the hashtag has never before 


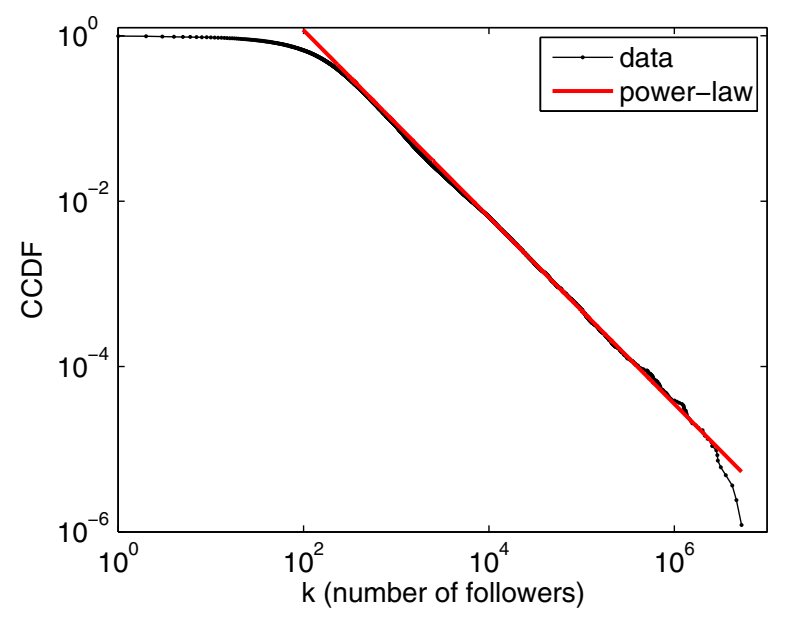

FIG. 5. CCDF for the number of followers $k$ of a random sample of $8.2 \times 10^{5}$ Twitter users. The straight line corresponds to an out-degree distribution with tail scaling as $p_{k} \sim D k^{-\gamma}$ as $k \rightarrow \infty$, with $D=240$ and $\gamma=2.13\left(x_{\min }=1.1 \times 10^{4}\right.$, fitted as described in Ref. [66]).

appeared in the system. Each innovation event thus increases the number of distinct hashtags that appear in the data set by 1 , whereas a noninnovative (copying) tweet will instead increase the number of copies of a hashtag that is already present in the data set. We can therefore calculate an upper bound on the empirical innovation probability from the ratio

$$
\begin{aligned}
\tilde{\mu} & =\frac{\text { number of distinct hashtags used in the data set }}{\text { total number of hashtags tweeted by users }} \\
& =\frac{322799}{5886837}=0.055
\end{aligned}
$$

Note that this upper bound is consistent with the parameter value of $\mu=0.033$ that is fitted in Fig. 6. The reason why Eq. (53) gives an upper bound rather than an exact value for $\mu$ is the finite size of the data set: The data collection started at a specific point in time; thus, any hashtags that are in fact copied from tweets received prior to the start date will be erroneously counted as "distinct hashtags" in the estimate, thus leading to an overestimate of the true innovation probability.

\section{B. Results using identical user activity rates}

Using the empirical Twitter out-degree distribution $p_{k}$, we apply the analytical results of Eqs. (40) and (A11) (which assume $\beta_{j k} \equiv 1$ ) to fit the model to the data in Fig. 6. Figures 6(a) and 6(b) show that the model-predicted age-dependent popularity distributions match reasonably well to the data, and Fig. 6(c) shows that the age-dependent mean can be fitted very closely by the model. The data
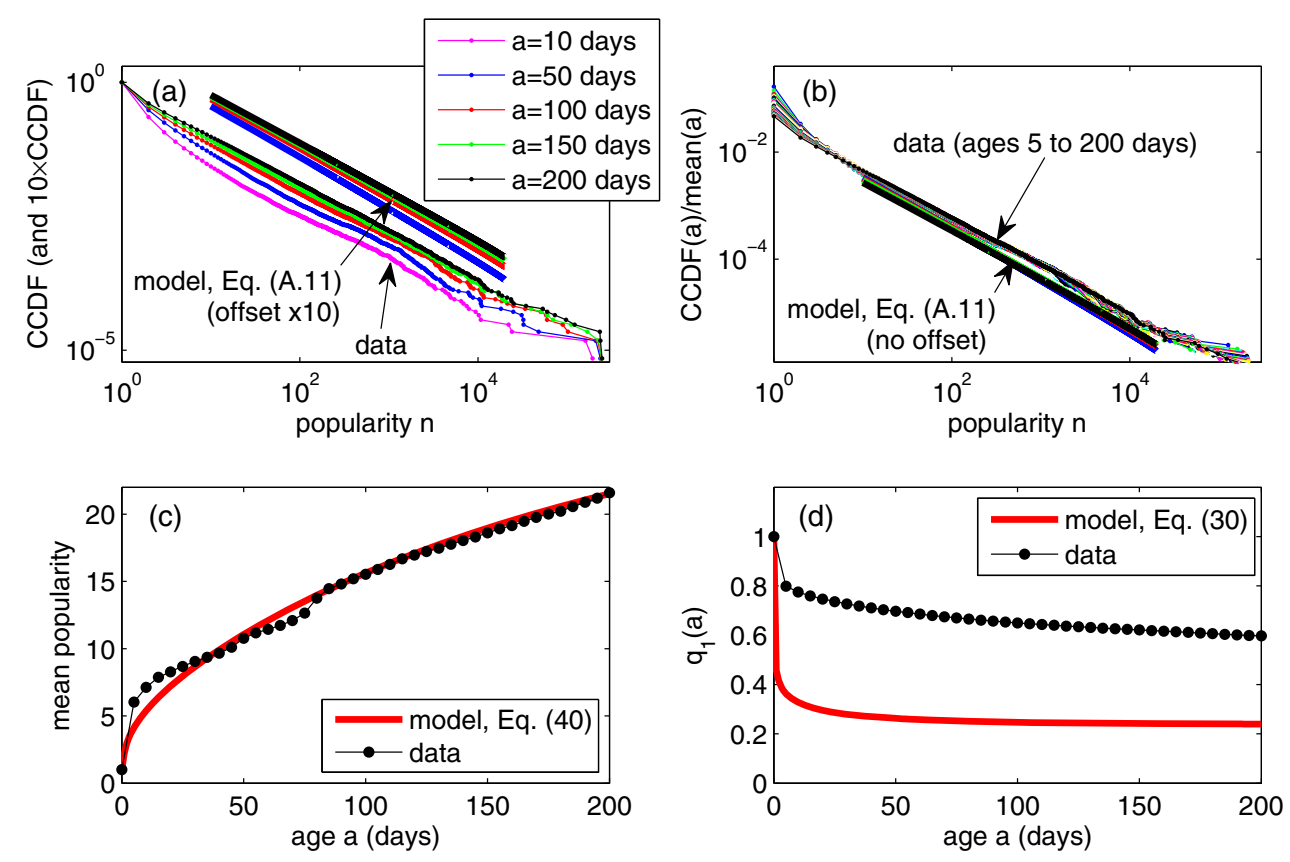

FIG. 6. Comparison of the model with Twitter hashtags data. (a) CCDFs for popularity of hashtags at age $a$ (at time $a$ after their first appearance in the data set). The model CCDFs [from Eq. (A11)] are multiplied by 10 for clarity. Model parameters are $\lambda=4.5 \times 10^{-4}$, $\mu=0.033, k_{G}=0.25, \theta=500$, with one model time unit corresponding to 0.16 days. (b) CCDFs at age $a$, each divided by the mean popularity at age $a$. The data show an apparent collapse onto a single curve that is closely matched by the model. (c) The mean popularity of hashtags of age $a$. (d) The fraction $q_{1}(a)$ of hashtags that are not retweeted by age $a$. Here, our basic model with homogeneous user activity rates does not fit well to the data (but see Fig. 7). 
collapse seen in Fig. 6(b) is intriguing, and we analyze it further in Sec. VIC.

Despite these successes, it was not possible to successfully fit the $q_{1}(a)$ curve [Fig. 6(d)] using the simplified version of the model in which all users have the same activity rates. In Sec. VID below, we therefore investigate the effect of heterogeneous activity rates and show that an improved fit can be obtained using more realistic rates.

\section{Analysis of the data collapse in Fig. 6(b)}

As shown in Fig. 6(b), the ratio $q_{n}(a) / m(a)$ is approximately independent of the age $a$, giving a collapse of the popularity distribution data (and of the model predictions) onto a single curve. As in Sec. IV E, the large- $n$ asymptotics of the popularity distribution are found from the small- $w$ expansion (with $w=1-x$ ) of $h(a ; x)=$ $1-H(a ; x)$, and for the scale-free out-degree distribution, we obtain from Eq. (A10) (using the final value theorem for Laplace transforms) the following asymptotic behavior in the $a \rightarrow \infty$ limit:

$$
h(\infty ; 1-w) \sim(\lambda z+1) C^{-[1 /(\gamma-1)]} w^{[1 /(\gamma-1)]} \quad \text { as } w \rightarrow 0
$$

Understanding the large- $a$ approach to this steady state (i.e., the case where $a$ is large but finite) is a difficult problem in asymptotic analysis, involving the double limits $n \rightarrow \infty$ and $a \rightarrow \infty$. However, some insight can be obtained by factoring the function $h$ into a product of its infinite-age limit $h(\infty ; x)$ and another function $h_{1}$, with $h_{1}$ limiting to 1 as $a \rightarrow \infty$ :

$$
h(a ; x)=h(\infty ; x) h_{1}(a ; x) .
$$

Taking Laplace transforms gives

$$
\hat{h}(s ; x)=h(\infty ; x) \hat{h}_{1}(s ; x),
$$

where

$$
\hat{h}_{1}(s ; 1-w)=\frac{\lambda z(s+\lambda z+\hat{\Phi}(s))}{s(\lambda z+1)(s+\lambda z)} \frac{(\gamma-1) \lambda D^{[1 /(\gamma-1)]}[\Gamma(1-\gamma)]^{[1 /(\gamma-1)]} w^{[(\gamma-2) /(\gamma-1)]} \hat{\Phi}(s)}{s+\lambda z-\lambda z \hat{\Phi}(s)+(\gamma-1) \lambda D^{[1 /(\gamma-1)]}[\Gamma(1-\gamma)]^{[1 /(\gamma-1)]} w^{[(\gamma-2) /(\gamma-1)]} \hat{\Phi}(s)} .
$$

In particular, note that $\hat{h}_{1}(s ; 1-w)$ depends on $w$ only through the factor $w^{[(\gamma-2) /(\gamma-1)]}$. In the case where $\gamma$ is very close to 2 , the exponent $(\gamma-2) /(\gamma-1)$ of the $w$ dependence is close to zero, and the dependence of $h_{1}$ on $w$ is therefore very weak. It follows that the rate of approach of the corresponding distribution $q_{n}(a)$ to the steady state $q_{n}(\infty)$ does not show a strong dependence on $n$, and the CCDFs for various ages appear almost parallel in the loglog plot of Fig. 6(a) (note $\gamma=2.13$ in the Twitter network).

As we saw in Sec. IV C for the large-age asymptotics of the mean popularity, the long-time behavior of the popularity distribution may be obtained by examining the linear (early-age) growth of the inverse transform of Eq. (A11). The resulting popularity distributions $q_{n}(a)$ show (for large $n)$ a regime of linear-in-age growth, and in the case where $\gamma \approx 2$, the rate of this growth depends only weakly on $n$. Since the mean popularity $m(a)$ is also growing linearly during this age period [see Eq. (43)], the division of the $\mathrm{CCDFs}$ at various ages by the corresponding mean $m(a)$ leads to the collapse of the data onto the single curve that is seen in Fig. 6(b).

\section{Heterogeneous activity rates}

Although our analysis methods are quite general, in order to focus on understanding the combined effects of memory and out-degree distribution, most of our results thus far are specialized to the case of uniform user activity rates, $\beta_{j k} \equiv 1$. It is interesting, therefore, to examine the impact that more realistic heterogeneous activity rates would have upon the results we have obtained. To this end, we extend here to the case where the activity rate of a user depends on its out-degree $k$ while retaining the assumption $p_{j k}=\delta_{j, z} p_{k}$, so that $\beta_{j k}=\beta_{k}$ (normalized so that $\sum_{k} \beta_{k} p_{k}=1$ and with $\bar{\beta}=\sum_{k} \frac{k}{z} \beta_{k} p_{k}$ ).

The mean popularity is given in the general case by Eq. (36). Repeating the asymptotic analysis leading to Eq. (43) for the $\mu \rightarrow 0$ limit, we again find linear growth of $m(a)$ with age $a$, with a slope that generalizes that found in Eq. (43):

$$
m(a) \sim \frac{\lambda z \bar{\beta}+\frac{\overline{\beta^{2}}}{\bar{\beta}}}{T \lambda z \bar{\beta}+1} a \quad \text { as } a \rightarrow \infty
$$

where we have introduced the notation $\overline{\beta^{2}} \equiv \sum_{k} \frac{k}{z}\left(\beta_{k}\right)^{2} p_{k}$.

If we additionally assume that the user activity rates saturate to a constant level $\beta_{\infty}$ at very large $k$, so $\beta_{k} \rightarrow \beta_{\infty}$ as $k \rightarrow \infty$, then we can repeat the asymptotic approximations of Sec. IVE to determine a generalized version of Eq. (A11): 

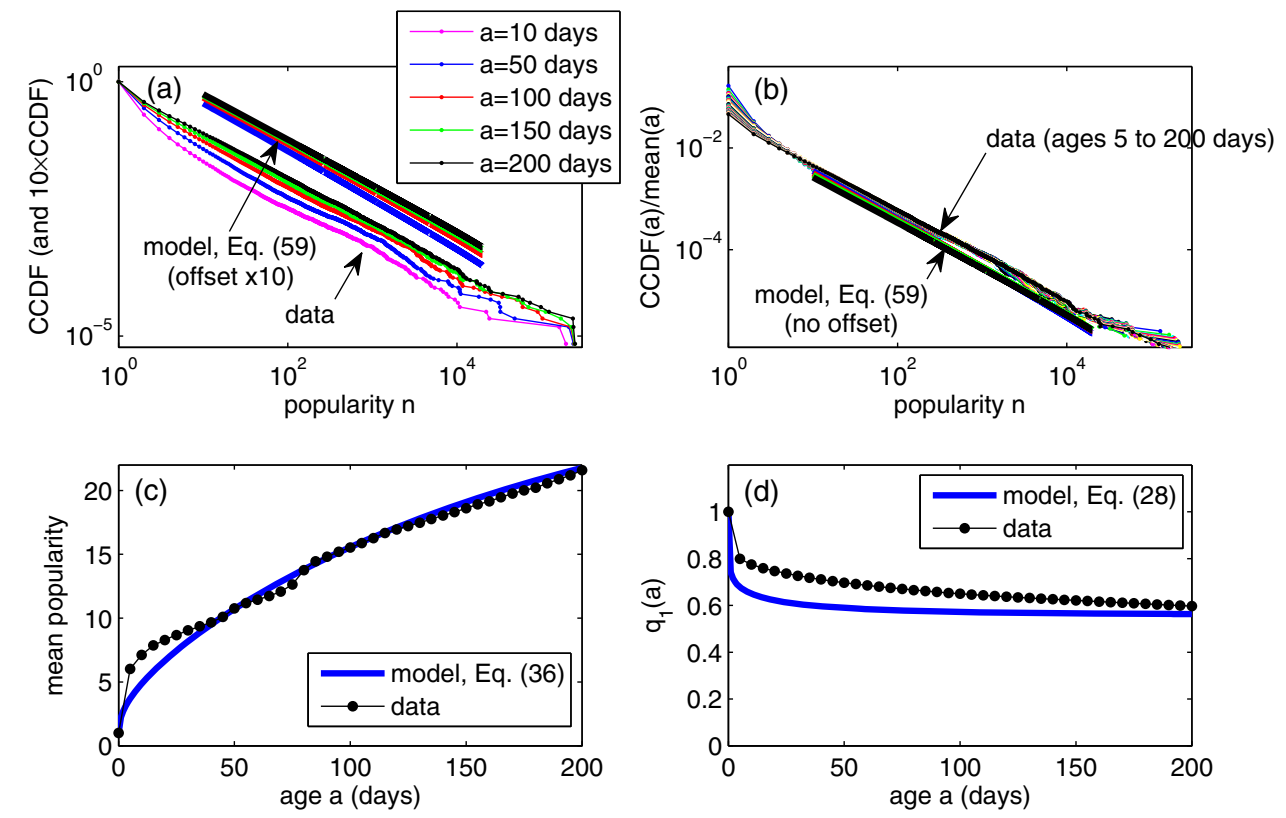

FIG. 7. Same as in Fig. 6 , but including heterogeneous activity rates $\beta_{k}$ given by Eq. (C1). Model parameters are $\lambda=5 \times 10^{-4}$, $\mu=0.033, k_{G}=0.25, \theta=500$, with one model time unit corresponding to 0.18 days.

$$
\hat{H}(s ; x) \sim \frac{1}{s}-\frac{1}{s} \frac{\lambda z \bar{\beta}\left(s+\lambda z \bar{\beta}+\frac{\overline{\beta^{2}}}{\bar{\beta}} \hat{\Phi}(s)\right)(\gamma-1)(1-x) \hat{\Phi}(s)}{(s+\lambda z \bar{\beta})\left(s+\lambda z \bar{\beta}-\lambda z \bar{\beta} \hat{\Phi}(s)+\beta_{\infty}^{[1 /(\gamma-1)]}(\gamma-1) \lambda D^{[1 /(\gamma-1)]}[\Gamma(1-\gamma)]^{[1 /(\gamma-1)]}(1-x)^{[(\gamma-2) /(\gamma-1)]} \hat{\Phi}(s)\right)} .
$$

To demonstrate the effect of heterogeneous activity rates, we consider a model for $\beta_{k}$ inspired by the data analysis shown in Fig. 6(a) of Ref. [38] (see Appendix C for details). Using this heterogeneous activity rate, Fig. 7 shows results that correspond closely to the homogeneous-activity example of Fig. 6. A comparison of panels (d) from both figures clearly shows that including heterogeneous activity rates leads to a better fit of the model to the data on the fraction $q_{1}(a)$ of nonretweeted memes. However, the other results of the model [panels (a)-(c) of Fig. 7 compared to the same panels in Fig. 6] are relatively unaffected by the activity rate, so the good matches between model and data seen in Fig. 6 are not compromised by including heterogeneity in activity rates.

\section{LIMITATIONS OF THE MODEL}

As we have demonstrated, the analytical tractability of the null model enables it to be fitted to time-dependent data on meme popularity. However, we were required to make a number of assumptions to obtain analytical results, and in this section, we briefly highlight the most important assumptions and discuss possible extensions to the model.

The network structure is assumed to be that of a directed configuration-model graph defined by the joint probability $p_{j k}$ of a node having in-degree $j$ and out-degree (number of followers) $k$. While this joint probability can encode correlations between the number followed by, and the number of followers of, a node, it does not incorporate edge-based correlations, i.e., the probability that a user with many followers is followed by users who also have high numbers of followers. It may be possible to extend the analysis of the model to deal with at least some types of edge correlation $[67,68]$, but this would be at the cost of increased complexity of the equations.

A more unrealistic simplification of the configuration model is the fact that it generates networks that are locally treelike, with few short cycles. In particular, our model does not include bidirectional edges (i.e., reciprocated following relationships, where user $A$ follows user $B$ and $B$ also follows $A$ ), which are quite common in the Twitter network [69] but which violate the independence assumption of a branching process. However, numerical simulations in Ref. [37] using a real Twitter network for a zero-memory version of the model (Sec. S4 of Ref. [37]) gave quite good agreement with branching process theory, despite the presence of a large fraction of reciprocal links in the graph. The conditions under which tree-based theories give good approximations for dynamics on nontreelike networks remains an active area of research [70], and more work is required for further understanding. 
An important assumption of the null model is that all memes have equal fitness. This is consistent with randomcopying models of human decision making [36,71], where the quality of the product-here, the "interestingness" of the meme-is less important than the social influence of peers' decisions [72]. This neutrality of the model is at the root of the criticality of the dynamical system [39]. A related (discrete-time) model for the number of citations gained by scientific papers was analyzed in Ref. [19], where the authors also extended their neutral model to include unequal fitnesses of papers. It is likely that our model could be extended in a similar way to incorporate a fitness parameter for each individual meme. Based on the results of Ref. [19], we expect that our main results would be qualitatively unaffected if the distribution of fitness values over the set of all memes is strongly peaked (i.e., if most memes have roughly equal fitness values, with only the high-fitness outliers demonstrating supercritical popularity growth).

Perhaps the most unrealistic aspect of the current model is the assumption that all users have constant activity rates, so their tweeting activity is described by a Poisson process (see the discussion in Sec. III B). It would be interesting to relax this assumption, for example, to allow the activity of users to be described by models such as that of Ref. [73] or by inhomogeneous Poisson processes: The latter incorporates time-varying activity rates and thus could model the 24-hour variability in tweeting levels determined by daily patterns [30]. However, we believe that the near-critical aspect of the model will not be strongly affected by such generalizations. To see this, consider the heuristic derivation of the branching number $\xi$ that was described at the end of Sec. IV A. Over a sufficiently long time window $W$, the expected number of interesting memes received into the stream of a $(j, k)$-class user is linear in the number $j$ of users followed, and this remains true even for inhomogeneous Poisson (or even non-Poisson) activities, provided that the observation window is long enough (e.g., such that the average rate $\bar{\beta}$ of incoming tweets should yield approximately similar values when time averaged over disjoint time windows of length $W$ ). Similarly, the expected number of retweets by the user during the time window can be written as in Eq. (23) but with the Poisson rate $\beta_{j k}$ replaced by its time-averaged value. The calculations of Eq. (24) then proceed as before, leading to the conclusion that the branching number limits to the critical value of 1 as $\mu \rightarrow 0$, which implies that non-Poisson user activity rates (or burstiness) will not affect the criticality of the model, which is a long-time (i.e., ages of memes limited to infinity) characteristic. Of course, the short-term behavior of the model [such as the small- $a$ behavior in panels (b)-(e) of Fig. 4] would be affected by introducing burstiness; incorporating such realistic features into the model is left as a challenge for further work. As a final comment on this topic, we note that the agreement (in Sec. VI) of our theoretical results with real data of a spreading process for which users' activity rates are not constant also provides indirect evidence that the phenomenology discussed is robust to the details of user activity burstiness.

The heuristic calculation of the branching number considered at the end of Sec. IVA also offers a clue as to how the model can be extended to the spreading of information on undirected social networks (as opposed to the directed networks that we focus on in this paper). Of particular interest is the spreading of app adoption on Facebook, for which data were analyzed in Ref. [10] and a computational model was introduced in Ref. [5]. If the Facebook update messages that inform all friends of user $A$ that she has installed a particular app are considered to be the memes in a version of our model, then the arguments of Sec. IV A need only slight modifications. The total number of update messages received in the stream of a user with $k$ Facebook friends is linear in $k$ [i.e., the $j$ in the denominator of Eq. (22) is replaced by $k$ ], while the expected number of friends who would be interested in user $A$ 's adoption of the app is $\lambda(k-1)$ (since one friend out of $k$ must have adopted it before $A$ in order to have spread the message to her). Following very similar steps to calculate the expected number $\xi$ of children of a meme-see the calculations leading to Eq. (24) —we find that

$$
\begin{aligned}
\xi_{\text {undirected }} & \rightarrow \sum_{k} \frac{k}{z} p_{k} \frac{\beta_{k} \lambda(k-1)}{k \bar{\beta} \lambda} \quad \text { as } \mu \rightarrow 0 \\
& =1-\frac{1}{z}
\end{aligned}
$$

Although this branching number is less than 1 , the mean number $z$ of friends on Facebook is large (e.g., Ref. [74] calculated $z \approx 190$ ), so $\xi_{\text {undirected }}$ is in fact very close to unity, implying that the information-spread process is close to criticality. Such a near-critical branching process was hypothesized in Ref. [5] to explain the observed fat-tailed distributions of app popularity in Facebook data and the temporal characteristics of the adoption behavior. The cascade sizes for other forms of "meme" spreading on Facebook have also been observed to have fat-tailed distributions [14]. Other undirected networks to which the model should be applicable include YouTube [63] and Digg [2,75].

Finally, our focus here has been on the statistical physics of the model, but for completeness, we should note the difficulties inherent in applying the model to data sets where memes may not be as simple to recognize and track as hashtags are. In Ref. [21], for example, the process of extracting memes (representing popular scientific terms) from data (citation archives of scientific publications) is explained in detail, and such effort will generally be required to identify and track the memes to which this null model might be applied. 
A related question is whether the popularity of online memes has any implications in terms of mass social movements in the offline world. This is a complex question that lies beyond the scope of this paper, but we note that Fig. 3 of Ref. [76] shows that the usage of hashtags related to the $15 \mathrm{M}$ Spanish protest movement was found to be closely correlated with the number of protest-related headlines in newspapers, at least during the main activity of the protests. This indicates that online social spreading phenomena can, at least in some cases, give useful information about real-world social movements and activism.

\section{DISCUSSION}

The extremely wide range of popularities achieved by items on social media poses many challenges for complex systems researchers. These include the identification of the causes [77] and structural features [78] of "viral" propagation, and the prediction of future spreading based on the content or the early-time growth of memes [4,11,16,79], each of which is important in the design of more efficient systems to spread information (e.g., in case of emergency). We argue that null models are fundamentally important in this quest—and complement more data-driven approaches-as they demonstrate, for example, that extreme popularity can arise purely because of random fluctuations in the competition between memes for user attention. While the content of a meme may well be an important factor in its popularity (or predictability [11]), definitive statements about the significance of such factors should be referenced to an appropriate null model.

In this paper, we have introduced and analyzed a null model of meme spreading that is analytically tractable yet realistic enough to reproduce several characteristic features of empirical data. The model is sufficiently general to incorporate heterogeneous user activity rates and a joint distribution $p_{j k}$ of the number of users followed $j$ and the number of followers $k$, as well as a memory-time distribution $\Phi$ that gives non-Markovian dynamics. The competition-induced criticality phenomenon identified in a zero-memory model in Ref. [37] is found to be robust to the generalizations, giving power-law popularity distributions with characteristic time dependence similar to data from social spreading phenomena (see Sec. VII for a discussion of further possible extensions of the model).

The analytical tractability enables fast fitting of the model to data, as demonstrated in Sec. VI with hashtag data from Twitter. We find that a simplified version of the model where users all have the same activity rate can be fitted to some, but not all, aspects of the data (see Fig. 6). The aim of a null model is not to perfectly reproduce every aspect of a data set but rather to help identify which features of the data can be reproduced using relatively simple models, thus to highlight aspects where more detailed modeling (or, perhaps, factors entirely outside the model) are required to match to data. In this respect, the null model highlights the fact that heterogeneity in activity rates is vital to accurately capture the $q_{1}(a)$ curve [compare Figs. 6(d) and $7(\mathrm{~d})$ ], even though the time dependence of the bulk of the popularity distribution may be described reasonably well by a model with homogeneous activity rates [Figs. 6(a)-6(c)].

As noted in the Introduction, and expanded upon in Sec. VII, our definition of "memes" is sufficiently general to enable the model to be applied (with minor changes) not just to the spreading of hashtags or URLs on Twitter but also to the adoption of apps on Facebook, the popularity of videos on YouTube, and to a broad range of imitationdriven spreading dynamics. We anticipate that the analytical results and potential for fast fitting to data will make this null model a useful tool for further work, and we hope it will contribute to the ongoing investigation of the entangled effects of memory, network structure, and competition on social spreading phenomena.

\section{ACKNOWLEDGMENTS}

The authors acknowledge helpful feedback from Davide Cellai, Timothy Duff, Rick Durrett, Freja Elbro, Ali Faqeeh, Peter Fennell, Kristina Lerman, David O'Sullivan, Mason Porter, and Jonathan Ward. This work was supported by Science Foundation Ireland [Grants No. 11/PI/1026, No. 12/ IA/1683, and No. 09/SRC/E1780 (J. P. G. and K. P.O'S.)] and by the European Commission through FET-Proactive projects PLEXMATH [FP7-ICT-2011-8; Grant No. 317614 (J. P.G and Y.M.)] and MULTIPLEX [FP7-ICT-2011-8; Grant No. 317532 (Y. M.)]. R. A. B. and Y. M. also acknowledge support from MINECO (Grant No. FIS2011-25167) and Comunidad de Aragón (Spain; Grant FENOL). We acknowledge the SFI/HEA Irish Centre for High-End Computing (ICHEC) for the provision of computational facilities. The data used in this paper are available for download from www.ul.ie/gleesonj/twitter15M.

\section{APPENDIX A: CALCULATION OF THE LARGE- $a$, LARGE- $n$ ASYMPTOTICS OF POPULARITY DISTRIBUTION}

In this appendix, we consider how the popularity distribution $q_{n}(a)$ behaves for large, but finite ages. To highlight the effect of the out-degree distribution $p_{k}$ upon the results, here we restrict our analysis to the case $\beta_{j k} \equiv 1$, $p_{j k}=\delta_{j, z} p_{k}$. Taking the $\mu \rightarrow 0$ limit, Eq. (15) becomes

$$
\begin{aligned}
G(a ; x)= & \sum_{k} p_{k} \int_{0}^{\infty} d \ell \lambda z e^{-\lambda z \ell} \\
& \times \exp \left[-\int_{0}^{\min (\ell, a)} d \tilde{r} \int_{0}^{a-\tilde{r}} d \tilde{\tau} \Phi(a-\tilde{r}-\tilde{\tau})\right. \\
& \left.\times\left(1-x[1-\lambda+\lambda G(\tilde{\tau} ; x)]^{k}\right)\right]
\end{aligned}
$$


Writing $x=1-w$ and $G(a ; x)=1-\phi(a ; w)$, we observe that the argument of the exponential function vanishes when $w=0$ and $\phi=0$, and so we consider the small-w, small- $\phi$ asymptotic behavior by expanding the exponential term to first order in its argument:

$$
\begin{aligned}
\phi(a ; w) \approx & \int_{0}^{\infty} d \ell \lambda z e^{-\lambda z \ell} \int_{0}^{\min (\ell, a)} d \tilde{r} \\
& \times \int_{0}^{a-\tilde{r}} d \tilde{\tau} \Phi(a-\tilde{r}-\tilde{\tau}) \\
& \times\left(1-(1-w) \sum_{k} p_{k}[1-\lambda \phi(\tilde{\tau} ; w)]^{k}\right) .
\end{aligned}
$$

We note that retaining only the first-order term in the expansion of the exponential is an approximation. We estimate the accuracy of this "one-term expansion" by comparing the infinite-age limit determined under the approximation with the corresponding exact values as given in Sec. IV D.

For the case of a scale-free out-degree distribution with $p_{k} \sim D k^{-\gamma}$ as $k \rightarrow \infty$, and $\gamma$ in the range $2<\gamma<3$, the asymptotic form of the summation term in Eq. (A2) is given by [37]

$$
\begin{gathered}
1-(1-w) \sum_{k} p_{k}[1-\lambda \phi]^{k} \sim \lambda z \phi-C \phi^{\gamma-1} \\
+w+o(w, \phi) \quad \text { as } w \rightarrow 0, \phi \rightarrow 0
\end{gathered}
$$

with the constant $C$ given by $C=\lambda^{\gamma-1} D \Gamma(1-\gamma)$. Applying the integral-swapping trick of Eq. (34) allows the righthand side of Eq. (A2) to be expressed as a double convolution integral. Laplace transforming then yields

$$
\hat{\phi}(s ; w)=\frac{1}{\lambda z+s} \hat{\Phi}(s) \mathcal{L}\left[\lambda z \phi-C \phi^{\gamma-1}+w\right],
$$

where $\mathcal{L}$ denotes the Laplace transform operation applied to the term in square brackets. In the $a \rightarrow \infty$ limit, this equation is satisfied by the steady-state solution

$$
\phi(\infty ; w)=C^{-[1 /(\gamma-1)]} w^{[1 /(\gamma-1)]}
$$

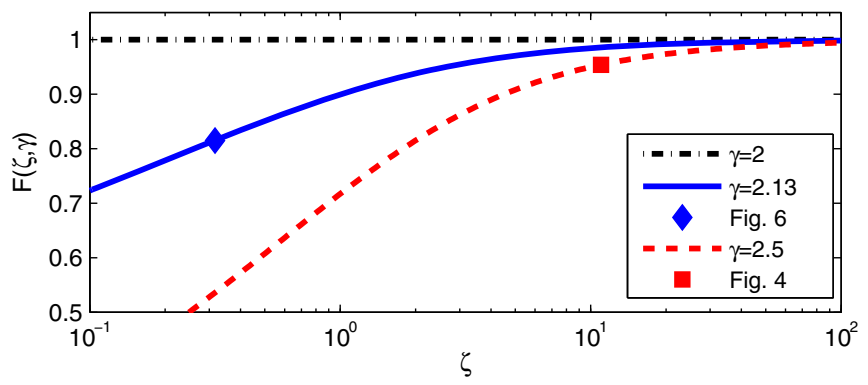

FIG. 8. The function $F(\zeta, \gamma)$, as defined in Eq. (A6), for values of $\gamma$ close to 2 . The highlighted points are the parameter values that are relevant to Fig. $4(\lambda z=11, \gamma=2.5)$ and to Fig. 6 $(\lambda z=0.32, \gamma=2.13)$. In all cases of interest, the values of $F$ are close to 1 , so we conclude that the one-term expansion used in Eq. (A2) gives a good estimate of the exact steady-state solution.

as can be verified using the final value theorem for Laplace transforms. We note that the corresponding expression for $\phi(\infty ; w)$ as calculated from the steady state Eq. (46) has an additional multiplicative factor of $F(\lambda z, \gamma)$ that is absent in Eq. (A5), where the function $F(\zeta, \gamma)$ is defined by

$$
F(\zeta, \gamma)=\left[\zeta^{2} \sum_{n=1}^{\infty} \frac{n^{\gamma-1}}{(\zeta+1)^{n+1}}\right]^{-[1 /(\gamma-1)]}
$$

(see Fig. 8). If $\lambda z \gg 1$, then $F(\lambda z, \gamma) \approx 1$, and the one-term expansion gives results that are very close to the exact values (at least in the infinite-age limit $a \rightarrow \infty$ ). Moreover, even if $\lambda z$ is not large (e.g., $\lambda z=0.32$ for the model fit to Twitter hashtags data in Sec. VI), the values of $F(\lambda z, \gamma)$ can still be close to unity if $\gamma$ is sufficiently close to 2 .

To consider small deviations from the steady state, we define $g(a ; w)$ by

$$
\phi(a ; w)=\phi(\infty ; w)(1-g(a ; w)),
$$

with $g(a ; w) \rightarrow 0$ as $a \rightarrow \infty$. Assuming that $g$ is sufficiently small to allow the use of the linearizing approximation

$$
(1-g)^{\gamma-1} \approx 1-(\gamma-1) g
$$

Eq. (A4) can be solved for the Laplace transform of $g$ :

$$
\hat{g}(s ; w)=\frac{1}{s} \frac{s+\lambda z-\lambda z \hat{\Phi}(s)}{s+\lambda z-\lambda z \hat{\Phi}(s)+(\gamma-1) C^{[1 /(\gamma-1)]} w^{[(\gamma-2) /(\gamma-1)]} \hat{\Phi}(s)} .
$$

The Laplace transform of $\phi$ then follows from Eq. (A7), and a similar asymptotic analysis of Eq. (17) yields

$$
\hat{H}(s ; 1-w)=\frac{1}{s}-\frac{\lambda z(s+\lambda z+\hat{\Phi}(s))}{s+\lambda z} \hat{\phi}(s ; w) .
$$

Substituting from Eqs. (A7) and (A9) results in 


$$
\hat{H}(s ; x)=\frac{1}{s}\left[1-\frac{\lambda z(s+\lambda z+\hat{\Phi}(s))(\gamma-1)(1-x) \hat{\Phi}(s)}{(s+\lambda z)\left(s+\lambda z-\lambda z \hat{\Phi}(s)+(\gamma-1) \lambda D^{[1 /(\gamma-1)]}[\Gamma(1-\gamma)]^{[1 /(\gamma-1)]}(1-x)^{[(\gamma-2) /(\gamma-1)]} \hat{\Phi}(s)\right)}\right] .
$$

A similar analysis can be performed in the case where the out-degree distribution $p_{k}$ has a finite second moment. We again utilize a one-term expansion similar to Eq. (A2), but we can also retain a nonvanishing innovation probability $\mu$ in this case. The one-term expansion can be shown to be accurate when $\lambda z \gg 1$; this condition is obeyed in all relevant cases we examine. The resulting large- $a$ asymptotics for the generating function $H(a ; x)$ are found by inverting the following Laplace transform:

$\hat{H}(s ; 1-w)=\frac{1}{s}-\phi(\infty ; w) \frac{(1-\mu) \lambda z(s+\lambda z+\mu+\hat{\Phi}(s))}{s(s+\lambda z+\mu)}\left[\frac{2(1-\mu) \frac{w}{\phi(\infty ; w)} \hat{\Phi}(s)-\mu(\lambda z+1) \hat{\Phi}(s)}{s+\lambda z+\mu-(\lambda z(1+\mu)+2 \mu) \hat{\Phi}(s)+2(1-\mu) \frac{w}{\phi(\infty ; w)} \hat{\Phi}(s)}\right]$,

with $\phi(\infty ; w)$ given by

$$
\phi(\infty ; w)=\frac{-\mu(\lambda z+1)+\sqrt{\mu^{2}(\lambda z+1)^{2}+2 \lambda^{2}(1-\mu)^{2}\left(\left\langle k^{2}\right\rangle-z\right) w}}{\lambda^{2}(1-\mu)\left(\left\langle k^{2}\right\rangle-z\right)} .
$$

\section{APPENDIX B: NUMERICAL INVERSION OF LAPLACE TRANSFORMS AND PGFS}

Many of our results for the popularity distribution $q_{n}(a)$ are expressed in terms of the corresponding PGF $H(a ; x)$. As in Ref. [37], we use the fast Fourier transform method of Refs. [46,80-82] to numerically invert the PGF at a fixed age $a$ to produce, for example, the model distributions in Figs. 6 and 7; see Sec. S2 of Ref. [37] for further details and links to Octave/Matlab code for implementing the PGF inversion.
The results of the model for the age dependence of several quantities are expressed in terms of Laplace transforms. To numerically invert the Laplace transforms, we use the efficient Talbot algorithm [83], in its simplified version described in Sec. 6 of Ref. [84]. The Talbot algorithm is based on a numerical evaluation of the Bromwich (Laplace inversion) integral, using a cleverly chosen deformation of the contour in the complex- $s$ plane. The Laplace inversion of $\hat{H}(s ; x)$ to obtain $H(a ; x)$ at a desired age $a$, for example, can be quickly computed using the $2 M_{L}-1$ weights $\gamma_{k}$ and nodes $\delta_{k}$ defined by [85]

$$
\begin{array}{ll}
\delta_{0}=\frac{2 M_{L}}{5}, & \delta_{k}=\frac{2 k \pi}{5}\left(\cot \left(k \pi / M_{L}\right)+i\right) \quad \text { for }-M_{L}+1 \leq k \leq M_{L}-1, \\
\gamma_{0}=\frac{1}{2} e^{\delta_{0}}, & \gamma_{k}=\left[1+i\left(k \pi / M_{L}\right)\left(1+\left[\cot \left(k \pi / M_{L}\right)^{2}\right]\right)-i \cot \left(k \pi / M_{L}\right)\right] e^{\delta_{k}} \quad \text { for }-M_{L}+1 \leq k \leq M_{L}-1
\end{array}
$$

(where $i=\sqrt{-1}$ ) by calculating the sum

$H(a ; x)=\frac{1}{5 a}\left[\gamma_{0} \hat{H}\left(\frac{\delta_{0}}{a} ; x\right)+\sum_{k=-M_{L}+1}^{M_{L}-1} \gamma_{k} \hat{H}\left(\frac{\delta_{k}}{a} ; x\right)\right]$.

In practice, the precision of the Talbot algorithm is very high, and only relatively small values of $M_{L}$ are required to obtain accurate results; we used $M_{L}=25$ in the examples shown.

\section{APPENDIX C: MODEL OF HETEROGENEOUS ACTIVITY RATES}

In the data analysis of Fig. 6(a) of Ref. [38], the average activity rate (as measured by the number of tweets by a user in a fixed time period) is found to grow approximately linearly with the number of followers $k$ of that user, for $k$ from 0 to about 100. Then, for $k$ values from about 100 up to the maximum shown in the plot $\left(k=10^{3}\right)$, the activity rate grows as a more slowly increasing linear function of $k$. We model these characteristics (which are also seen in other studies, e.g., Ref. [86]), using a piecewise-linear and 
continuous function of $k$, assuming a saturation of activity at very high $k$, as follows:

$\beta_{k} \propto \begin{cases}0.35 k & \text { if } k<100 \\ 35+0.044(k-100) & \text { if } 100 \leq k<10^{4} \\ 470.6 & \text { if } k \geq 10^{4},\end{cases}$

where the values are chosen to closely match the linear growth rates in Fig. 6(a) of Ref. [38], and with the constant of proportionality being set by the condition $\sum_{k} \beta_{k} p_{k}=1$.

[1] E. Bakshy, J. M. Hofman, W. A. Mason, and D. J. Watts, Everyone's an Influencer: Quantifying Influence on Twitter, in Proceedings of the Fourth ACM International Conference on Web Search and Data Mining (ACM, New York, 2011), pp. 65-74.

[2] K. Lerman, R. Ghosh, and T. Surachawala, Social Contagion: An Empirical Study of Information Spread on Digg and Twitter Follower Graphs, arXiv:1202.3162.

[3] L. Weng, A. Flammini, A. Vespignani, and F. Menczer, Competition Among Memes in a World with Limited Attention, Sci. Rep. 2, 335 (2012).

[4] J. Cheng, L. Adamic, P. A. Dow, J. M. Kleinberg, and J. Leskovec, Can Cascades Be Predicted?, in Proceedings of the 23rd International Conference on World Wide Web (ACM, New York, 2014), pp. 925-936.

[5] J. P. Gleeson, D.Cellai, J.-P. Onnela, M. A. Porter, and F. Reed-Tsochas, A Simple Generative Model of Collective Online Behavior, Proc. Natl. Acad. Sci. U.S.A. 111, 10411 (2014).

[6] C. Castellano, S. Fortunato, and V. Loreto, Statistical Physics of Social Dynamics, Rev. Mod. Phys. 81, 591 (2009).

[7] J. Leskovec, L. A. Adamic, and B. A. Huberman, The Dynamics of Viral Marketing, ACM Trans. Web 1, 5 (2007).

[8] R. Pastor-Satorras and A. Vespignani, Epidemic Spreading in Scale-Free Networks, Phys. Rev. Lett. 86, 3200 (2001).

[9] R. Pastor-Satorras, C. Castellano, P. Van Mieghem, and A. Vespignani, Epidemic Processes in Complex Networks, Rev. Mod. Phys. 87, 925 (2015).

[10] J.-P. Onnela and F. Reed-Tsochas, Spontaneous Emergence of Social Influence in Online Systems, Proc. Natl. Acad. Sci. U.S.A. 107, 18375 (2010).

[11] J. M. Miotto and E. G. Altmann, Predictability of Extreme Events in Social Media, PLoS One 9, e111506 (2014).

[12] R. Dawkins, The Selfish Gene (Oxford University Press, New York, 2006).

[13] http://oxforddictionaries.com/definition/english/meme.

[14] L. A. Adamic, T. M. Lento, E. Adar, and P. C. Ng, Information Evolution in Social Networks, arXiv:1402.6792.

[15] J. Leskovec, L. Backstrom, and J. Kleinberg, MemeTracking and the Dynamics of the News Cycle, in Proceedings of the 15th ACM SIGKDD International Conference on Knowledge Discovery and Data Mining (ACM, New York, 2009), pp. 497-506.
[16] L. Weng, F. Menczer, and Y.-Y. Ahn, Virality Prediction and Community Structure in Social Networks, Sci. Rep. 3, 2522 (2013).

[17] R. A. Bentley, M. W. Hahn, and S. J. Shennan, Random Drift and Culture Change, Proc. R. Soc. B 271, 1443 (2004).

[18] H. A. Herzog, R. A. Bentley, and M. W. Hahn, Random Drift and Large Shifts in Popularity of Dog Breeds, Proc. Roy. Soc. Lond. B Suppl. 271, S353 (2004).

[19] M. V. Simkin and V. P. Roychowdhury, A Mathematical Theory of Citing, J. Am. Soc. Inf. Sci. Tech. 58, 1661 (2007).

[20] S. Redner, How Popular Is Your Paper? An Empirical Study of the Citation Distribution, Eur. Phys. J. B 4, 131 (1998).

[21] T. Kuhn, M. Perc, and D. Helbing, Inheritance Patterns in Citation Networks Reveal Scientific Memes, Phys. Rev. X 4, 041036 (2014).

[22] D. de Solla Price, A General Theory of Bibliometric and Other Cumulative Advantage Processes, J. Am. Soc. Inf. Sci. 27, 292 (1976).

[23] M. E. J. Newman, Power Laws, Pareto Distributions and Zipf's Law, Contemp. Phys. 46, 323 (2005).

[24] M. Perc, The Matthew Effect in Empirical Data, J. R. Soc. Interface 11, 20140378 (2014).

[25] A.-L. Barabási and R. Albert, Emergence of Scaling in Random Networks, Science 286, 509 (1999).

[26] J. P. Sethna, K. A. Dahmen, and C. R. Myers, Crackling Noise, Nature (London) 410, 242 (2001).

[27] H. E. Stanley, Introduction to Phase Transitions and Critical Phenomena (Oxford University Press, New York, 1987).

[28] P. Bak, How Nature Works: The Science of Self-Organized Criticality (Springer, New York, 1999).

[29] A.-L. Barabasi, The Origin of Bursts and Heavy Tails in Human Dynamics, Nature (London) 435, 207 (2005).

[30] R. D. Malmgren, D. B. Stouffer, A. E. Motter, and L. A. N. Amaral, A Poissonian Explanation for Heavy Tails in EMail Communication, Proc. Natl. Acad. Sci. U.S.A. 105, 18153 (2008).

[31] M. Karsai, M. Kivelä, R. Kumar Pan, K. Kaski, J. Kertész, A.-L. Barabási, and J. Saramäki, Small but Slow World: How Network Topology and Burstiness Slow Down Spreading, Phys. Rev. E 83, 025102 (2011).

[32] J.-C. Delvenne, R. Lambiotte, and L. E. C. Rocha, Diffusion on Networked Systems Is a Question of Time or Structure, Nat. Commun. 6, 7366 (2015).

[33] H.-H. Jo, J. I. Perotti, K. Kaski, and J. Kertész, Analytically Solvable Model of Spreading Dynamics with Non-Poissonian Processes, Phys. Rev. X 4, 011041 (2014).

[34] J. L. Iribarren and E. Moro, Impact of Human Activity Patterns on the Dynamics of Information Diffusion, Phys. Rev. Lett. 103, 038702 (2009).

[35] J. L. Iribarren and E. Moro, Branching Dynamics of Viral Information Spreading, Phys. Rev. E 84, 046116 (2011).

[36] R. A. Bentley, P. Ormerod, and M. Batty, Evolving Social Influence in Large Populations, Behav. Ecol. Sociobiol. 65, 537 (2011).

[37] J. P. Gleeson, J. A. Ward, K. P. O’Sullivan, and W. T. Lee, Competition-Induced Criticality in a Model of Meme Popularity, Phys. Rev. Lett. 112, 048701 (2014). 
[38] N. O. Hodas, F. Kooti, and K. Lerman, Friendship Paradox Redux: Your Friends Are More Interesting Than You, arXiv:1304.3480.

[39] O. A. Pinto and M. A. Muñoz, Quasi-Neutral Theory of Epidemic Outbreaks, PLoS One 6, e21946 (2011).

[40] M. Kimura, The Neutral Theory of Molecular Evolution (Cambridge University Press, Cambridge, England, 1984).

[41] G. Bianconi and A.-L Barabási, Competition and Multiscaling in Evolving Networks, Europhys. Lett. 54, 436 (2001).

[42] M. V. Simkin and V. P. Roychowdhury, Re-inventing Willis, Phys. Rep. 502, 1 (2011).

[43] H. A. Simon, On a Class of Skew Distribution Functions, Biometrika 42, 425 (1955).

[44] T. E. Harris, The Theory of Branching Processes (Courier Corporation, 2002).

[45] H. S. Wilf, Generatingfunctionology (Elsevier, New York, 2013).

[46] M. E. J. Newman, S. H. Strogatz, and D. J. Watts, Random Graphs with Arbitrary Degree Distributions and Their Applications, Phys. Rev. E 64, 026118 (2001).

[47] K.-I. Goh, D.-S. Lee, B. Kahng, and D. Kim, Sandpile on Scale-Free Networks, Phys. Rev. Lett. 91, 148701 (2003).

[48] N. Schwartz, R. Cohen, D. Ben-Avraham, A.-L. Barabási, and S. Havlin, Percolation in Directed Scale-Free Networks, Phys. Rev. E 66, 015104 (2002).

[49] C. Adami and J. Chu, Critical and Near-Critical Branching Processes, Phys. Rev. E 66, 011907 (2002).

[50] S. Zapperi, K. B. Lauritsen, and H. E. Stanley, SelfOrganized Branching Processes: Mean-Field Theory for Avalanches, Phys. Rev. Lett. 75, 4071 (1995).

[51] N. O. Hodas and K. Lerman, How Visibility and Divided Attention Constrain Social Contagion, in Proceedings of the 2012 International Conference on Privacy, Security, Risk and Trust (PASSAT) and the 2012 International Conference on Social Computing (SocialCom) (IEEE, Amsterdam, 2012), pp. 249-257.

[52] M. E. J. Newman, The First-Mover Advantage in Scientific Publication, Europhys. Lett. 86, 68001 (2009).

[53] A. Vazquez, B. Racz, A. Lukacs, and A.-L. Barabasi, Impact of Non-Poissonian Activity Patterns on Spreading Processes, Phys. Rev. Lett. 98, 158702 (2007).

[54] B. Min, K.-I. Goh, and A. Vazquez, Spreading Dynamics Following Bursty Human Activity Patterns, Phys. Rev. E 83, 036102 (2011).

[55] P. Holme and J. Saramäki, Temporal Networks, Phys. Rep. 519, 97 (2012).

[56] T. Hoffmann, M. A Porter, and R. Lambiotte, Generalized Master Equations for Non-Poisson Dynamics on Networks, Phys. Rev. E 86, 046102 (2012).

[57] M. Boguná, L. F. Lafuerza, R. Toral, and M. Á. Serrano, Simulating Non-Markovian Stochastic Processes, Phys. Rev. E 90, 042108 (2014).

[58] P. Van Mieghem and R. Van de Bovenkamp, NonMarkovian Infection Spread Dramatically Alters the Susceptible-Infected-Susceptible Epidemic Threshold in Networks, Phys. Rev. Lett. 110, 108701 (2013).

[59] M. Kivelä and M. A. Porter, Estimating Interevent Time Distributions from Finite Observation Periods in Communication Networks, Phys. Rev. E 92, 052813 (2015).
[60] K. B. Athreya and P. E. Ney, Branching Processes, Vol. 196 (Springer Science \& Business Media, New York, 2012).

[61] C. Doerr, N. Blenn, and P. Van Mieghem, Lognormal Infection Times of Online Information Spread, PLoS One 8, e64349 (2013).

[62] R. A. Baños, J. Borge-Holthoefer, and Y. Moreno, The Role of Hidden Influentials in the Diffusion of Online Information Cascades, EPJ Data Science 2, 6 (2013).

[63] G. Szabo and B. A. Huberman, Predicting the Popularity of Online Content, Commun. ACM 53, 80 (2010).

[64] J. Borge-Holthoefer, A. Rivero, I. García, E. Cauhé, A. Ferrer, D. Ferrer, D. Francos, D. Iñiguez, M. P. Pérez, G. Ruiz et al., Structural and Dynamical Patterns on Online Social Networks: The Spanish May 15th Movement as a Case Study, PLoS One 6, e23883 (2011).

[65] S. González-Bailón, J. Borge-Holthoefer, A. Rivero, and Y. Moreno, The Dynamics of Protest Recruitment through an Online Network, Sci. Rep. 1, 197 (2011).

[66] A. Clauset, C. R. Shalizi, and M. E. J. Newman, Power-Law Distributions in Empirical Data, SIAM Rev. 51, 661 (2009).

[67] M. Boguñá and M. Á. Serrano, Generalized Percolation in Random Directed Networks, Phys. Rev. E 72, 016106 (2005).

[68] T. R. Hurd, The Construction and Properties of Assortative Configuration Graphs. arXiv:1512.03084.

[69] H. Kwak, C. Lee, H. Park, and S. Moon, What is Twitter, A Social Network or a News Media?, in Proceedings of the 19th International Conference on World Wide Web (ACM, New York, 2010), pp. 591-600.

[70] S. Melnik, A. Hackett, M. A. Porter, P. J. Mucha, and J. P. Gleeson, The Unreasonable Effectiveness of Tree-Based Theory for Networks with Clustering, Phys. Rev. E 83, 036112 (2011).

[71] A. Bentley, M. Earls, M. J. O'Brien, and J. Maeda, I'll Have What She's Having: Mapping Social Behavior (MIT Press, Cambridge, MA, 2011).

[72] M. J. Salganik, P. S. Dodds, and D. J. Watts, Experimental Study of Inequality and Unpredictability in an Artificial Cultural Market, Science 311, 854 (2006).

[73] N. Perra, B. Gonçalves, R. Pastor-Satorras, and A. Vespignani, Activity Driven Modeling of Time Varying Networks, Sci. Rep. 2, 469 (2012).

[74] J. Ugander, B. Karrer, L. Backstrom, and C. Marlow, The Anatomy of the Facebook Social Graph, arXiv:1111.4503.

[75] F. Wu and B. A. Huberman, Novelty and Collective Attention, Proc. Natl. Acad. Sci. U.S.A. 104, 17599 (2007).

[76] S. González-Bailón, J. Borge-Holthoefer, and Y. Moreno, Broadcasters and Hidden Influentials in Online Protest Diffusion, Am. Behav. Sci. 57, 943 (2013).

[77] M. Coscia, Average Is Boring: How Similarity Kills a Meme's Success, Sci. Rep. 4, 6477 (2014).

[78] S. Goel, A. Anderson, J. Hofman, and D. J. Watts, The Structural Virality of Online Diffusion, Management Science 62, 180 (2015).

[79] M. ten Thij, T. Ouboter, D. Worm, N. Litvak, H. Berg, and S. Bhulai, Algorithms and Models for the Web Graph: Proceedings of the 11th International Workshop, WAW 
2014, Beijing, China, 2014, Proceedings, Modelling of Trends in Twitter Using Retweet Graph Dynamics (Springer International Publishing, Switzerland, 2014), pp. 132-147.

[80] J. K. Cavers, On the Fast Fourier Transform Inversion of Probability Generating Functions, IMA J. Appl. Math. 22, 275 (1978).

[81] M. Marder, Dynamics of Epidemics on Random Networks, Phys. Rev. E 75, 066103 (2007).

[82] J. Abate and W. Whitt, Numerical Inversion of Probability Generating Functions, Operations Research Letters 12, 245 (1992).
[83] A. Talbot, The Accurate Numerical Inversion of Laplace Transforms, IMA J. Appl. Math. 23, 97 (1979).

[84] J. Abate and W. Whitt, A Unified Framework for Numerically Inverting Laplace Transforms, INFORMS J. Comput. 18, 408 (2006).

[85] J. Abate and P. P. Valkó, Multi-Precision Laplace Transform Inversion, Int. J. Numer. Meth. Engng. 60, 979 (2004).

[86] S. A. Myers and J. Leskovec, The Bursty Dynamics of the Twitter Information Network, in Proceedings of the 23rd International Conference on the World Wide Web (ACM, New York, 2014), pp. 913-924. 